\title{
FUTURE ASYMPTOTIC BEHAVIOUR OF TILTED BIANCHI MODELS OF TYPE IV AND VII ${ }_{h}$
}

\author{
SIGBJØRN HERVIK ${ }^{1}$, ROBERT VAN DEN HOOGEN ${ }^{2,1}$ AND ALAN COLEY ${ }^{1}$
}

\begin{abstract}
Using dynamical systems theory and a detailed numerical analysis, the late-time behaviour of tilting perfect fluid Bianchi models of types IV and $\mathrm{VII}_{h}$ are investigated. In particular, vacuum plane-wave spacetimes are studied and the important result that the only future attracting equilibrium points for noninflationary fluids are the plane-wave solutions in Bianchi type $\mathrm{VII}_{h}$ models is discussed. A tiny region of parameter space (the loophole) in the Bianchi type IV model is shown to contain a closed orbit which is found to act as an attractor (the Mussel attractor). From an extensive numerical analysis it is found that at late times the normalised energy-density tends to zero and the normalised variables 'freeze' into their asymptotic values. A detailed numerical analysis of the type $\mathrm{VII}_{h}$ models then shows that there is an open set of parameter space in which solution curves approach a compact surface that is topologically a torus.
\end{abstract}

\section{INTRODUCTION}

In recent years there has been much analysis of spatially homogeneous ( $\mathrm{SH}$ ) perfect fluid cosmological models with equation of state $p=(\gamma-1) \mu$, where $\gamma$ is constant [1]. For the class of tilted (non-orthogonal) SH models [2], the Einstein field equations have been written as an autonomous system of differential equations in a number of different ways 3, 4, 5. Tilted Bianchi models contain additional degrees of freedom, and new dynamical features emerge with the inclusion of tilt. A subclass of models of Bianchi type V [6, 7, 8, 9] and models of Bianchi type II [10] have been studied.

In [11] a local stability analysis of tilting Bianchi class $\mathrm{B}$ models and the type $\mathrm{VI}_{0}$ model of class $\mathrm{A}$ were studied. The asymptotic late-time behaviour of general tilted Bianchi type $\mathrm{VI}_{0}$ universes was analysed in detail in 12, and this was generalized to Bianchi type $\mathrm{VI}_{0}$ cosmological models containing two tilted $\gamma$-law perfect fluids in 13. Finally, the tilting Bianchi type $\mathrm{VI}_{h}$ and $\mathrm{VII}_{h}$ models were recently studied in 14 (self-similar tilting solutions [15] of Bianchi type $\mathrm{VI}_{h}$ were also studied in [16]).

In particular, the late-time behaviour of Bianchi models of type IV and $\mathrm{VII}_{h}$ was analysed in 14, with an emphasis on the stability properties of the vacuum plane-wave spacetimes. It was shown that for $2 / 3<\gamma<2$ there will always be future stable plane-wave solutions in the set of type IV and $\mathrm{VII}_{h}$ tilted Bianchi models and it was proven that the only future attracting equilibrium points for non-inflationary fluids $(\gamma>2 / 3)$ are the plane-wave solutions in Bianchi type $\mathrm{VII}_{h}$ models. A tiny region of the parameter space was discovered in the type IV model in which a closed orbit occurs. Moreover, for the type $\mathrm{VII}_{h}$ models a Hopf-bifurcation occurs and more attracting closed orbits appear.

In this paper we will use a dynamical systems approach to analyse the tilting Bianchi type IV and $\mathrm{VII}_{h}$ models in more detail, with an emphasis on numerical analysis. Since this current paper is a companion paper to [14, we shall not reproduce all of the details of the analysis and we shall frequently refer to equations in the original paper. However, we shall attempt to make the current paper as self-contained as possible.

We note that the Bianchi $\mathrm{VII}_{h}$ models are of special importance in cosmology. We recall that Bianchi type $\mathrm{VII}_{h}$ models are of maximal generality in the space of all SH models and are sufficiently general to account for many interesting phenomena. In particular, these cosmological models are the most general models that contain the open Friedmann-Robertson-Walker models and are consequently of special interest. Therefore,

Date: November 23, 2018. 
these anisotropic generalizations of the open Friedmann-Robertson-Walker models have been studied in detail in order to examine the dynamical behaviour of exact solutions of the Einstein field equation and, in particular, to study the evolution of large scale anisotropies in models that contain the standard low density universe as a limiting case.

There are a number of observational constraints on Bianchi models. For example, the structure of the Cosmic Microwave Background radiation anisotropy over intermediate scales can be used to constrain the open Bianchi type $\mathrm{VII}_{h}$ cosmological models 17. If the universe is open, then a distorted quadrupole or hotspot effect can occur [19]. However, the resulting constraint is of no practical use if the density lies close to the critical density (as suggested by recent observations). However, even if the density lies close to the critical density, in Bianchi $\mathrm{VII}_{h}$ models the anisotropic 3-curvature produces a general relativistic geodesic spiralling effect 20]. The spiral plus quadrupole are both focussed by the hotspot effect allowing a possible discrimination of the open Bianchi $\mathrm{VII}_{h}$ models.

The asymptotic and intermediate evolution of non-tilting perfect fluid Bianchi $\mathrm{VII}_{h}$ models has been studied using a detailed qualitative analysis [1, in conjunction with numerical experimentation [18. It is known that although Bianchi $\mathrm{VII}_{h}$ universes can isotropize at late times, the set of models that do so are of measure zero in the the class of all Bianchi models 20. The occurrence of intermediate isotropization in this class of non-tilting universes has been studied in [21] using approximate solutions of the Einstein field equations. More recently the evolution of the shear in Bianchi $\mathrm{VII}_{h}$ models was discussed heuristically 22]; in particular, it was claimed that if initial conditions at the Planck time consistent with the Planck equipartition principle are imposed, then the shear will decay sufficiently by the present epoch in order to be consistent with the low anisotropy in the Cosmic Microwave Background radiation. In 18 it was found that while intermediate isotropization may occur, it does not necessarily do so, and the approximate and heuristic analyses of 21] and 22] were critically examined. The linear stability of Bianchi type $\mathrm{VII}_{h}$ vacuum $^{2}$ plane-wave solutions has recently been studied in 23 .

The paper is organised as follows. First, we shall write down the equations of motion using the orthonormal frame formalism in the ' $N$-gauge'. Then, in section 3 we present all of the equilibrium points and investigate their stability properties. In section 4 we prove the existence of closed loops and provide some criteria for their stability. Then we will study the Bianchi type $\mathrm{VII}_{h}$ (and IV) models numerically in detail. Some of this numerical analysis is distributed throughout the paper; however, some explicit examples (e.g., the physically interesting case of dust and radiation) are given in Appendix A. In particular, using a detailed numerical analysis of the type $\mathrm{VII}_{h}$ models, we shall fully justify the claim in [14 that there is an open set of parameter space in which solution curves approach a compact surface that is topologically a torus.

\section{Equations of MOTION}

The companion paper 14] contains all the details regarding the determination of the evolution equations for the tilted cosmological models under consideration. These equations, written in gauge invariant form, allow one to choose the gauge that is best suited to the application at hand. Here, we shall adopt the $N$-gauge in which the function $\mathbf{N}_{\times}$is purely imaginary; this is ensured by the choice $\phi^{\prime}=\sqrt{3} \lambda \Sigma_{-}$, where $\lambda$ is defined by $\bar{N}=\lambda \operatorname{Im}\left(\mathbf{N}_{\times}\right)$. The evolution equation for $\bar{N}$ can then be replaced with an evolution equation for $\lambda$, which ensures a closed system of equations. We note that this choice of gauge is well suited for the study of the dynamics close to the vacuum plane-wave solutions 14. For a qualitative analysis of these models, the $N$-gauge is preferable since the resulting dynamical system is well defined in the Bianchi $\mathrm{VI}_{h}$ case. However, care must be exercised when analyzing the Bianchi $\mathrm{VII}_{h}$ case, in which the dynamical system is not well defined over the entire region of state space, in particular the variable $\lambda$ could diverge.

In the notation of [14, the expansion-normalised anisotropy and curvature variables used in this paper are

$$
\boldsymbol{\Sigma}_{1}=\Sigma_{12}+i \Sigma_{13}, \quad \boldsymbol{\Sigma}_{\times}=\Sigma_{-}+i \Sigma_{23}, \quad \mathbf{N}_{\times}=i N
$$


We will also adopt the dimensionless time parameter $\tau$, which is related to the cosmological time $t$ via $\mathrm{d} t / \mathrm{d} \tau=(1 / H)$, where $H$ is the Hubble scalar.

Using expansion-normalised variables, the equations of motion in the $N$-gauge are (see 14 for the complete derivation of the equations):

$$
\begin{aligned}
\Sigma_{+}^{\prime} & =(q-2) \Sigma_{+}+3\left(\Sigma_{12}^{2}+\Sigma_{13}^{2}\right)-2 N^{2}+\frac{\gamma \Omega}{2 G_{+}}\left(-2 v_{1}^{2}+v_{2}^{2}+v_{3}^{2}\right) \\
\Sigma_{-}^{\prime} & =\left(q-2-2 \sqrt{3} \Sigma_{23} \lambda\right) \Sigma_{-}+\sqrt{3}\left(\Sigma_{12}^{2}-\Sigma_{13}^{2}\right)+2 A N+\frac{\sqrt{3} \gamma \Omega}{2 G_{+}}\left(v_{2}^{2}-v_{3}^{2}\right) \\
\Sigma_{12}^{\prime} & =\left(q-2-3 \Sigma_{+}-\sqrt{3} \Sigma_{-}\right) \Sigma_{12}-\sqrt{3}\left(\Sigma_{23}+\Sigma_{-} \lambda\right) \Sigma_{13}+\frac{\sqrt{3} \gamma \Omega}{G_{+}} v_{1} v_{2} \\
\Sigma_{13}^{\prime} & =\left(q-2-3 \Sigma_{+}+\sqrt{3} \Sigma_{-}\right) \Sigma_{13}-\sqrt{3}\left(\Sigma_{23}-\Sigma_{-} \lambda\right) \Sigma_{12}+\frac{\sqrt{3} \gamma \Omega}{G_{+}} v_{1} v_{3} \\
\Sigma_{23}^{\prime} & =(q-2) \Sigma_{23}-2 \sqrt{3} N^{2} \lambda+2 \sqrt{3} \lambda \Sigma_{-}^{2}+2 \sqrt{3} \Sigma_{12} \Sigma_{13}+\frac{\sqrt{3} \gamma \Omega}{G_{+}} v_{2} v_{3} \\
N^{\prime} & =\left(q+2 \Sigma_{+}+2 \sqrt{3} \Sigma_{23} \lambda\right) N \\
\lambda^{\prime} & =2 \sqrt{3} \Sigma_{23}\left(1-\lambda^{2}\right) \\
A^{\prime} & =\left(q+2 \Sigma_{+}\right) A
\end{aligned}
$$

The equations for the fluids are:

$$
\begin{aligned}
\Omega^{\prime} & =\frac{\Omega}{G_{+}}\left\{2 q-(3 \gamma-2)+2 \gamma A v_{1}+[2 q(\gamma-1)-(2-\gamma)-\gamma \mathcal{S}] V^{2}\right\} \\
v_{1}^{\prime} & =\left(T+2 \Sigma_{+}\right) v_{1}-2 \sqrt{3} \Sigma_{13} v_{3}-2 \sqrt{3} \Sigma_{12} v_{2}-A\left(v_{2}^{2}+v_{3}^{2}\right)-\sqrt{3} N\left(v_{2}^{2}-v_{3}^{2}\right) \\
v_{2}^{\prime} & =\left(T-\Sigma_{+}-\sqrt{3} \Sigma_{-}\right) v_{2}-\sqrt{3}\left(\Sigma_{23}+\Sigma_{-} \lambda\right) v_{3}+\sqrt{3} \lambda N v_{1} v_{3}+(A+\sqrt{3} N) v_{1} v_{2} \\
v_{3}^{\prime} & =\left(T-\Sigma_{+}+\sqrt{3} \Sigma_{-}\right) v_{3}-\sqrt{3}\left(\Sigma_{23}-\Sigma_{-} \lambda\right) v_{2}-\sqrt{3} \lambda N v_{1} v_{2}+(A-\sqrt{3} N) v_{1} v_{3} \\
V^{\prime} & =\frac{V\left(1-V^{2}\right)}{1-(\gamma-1) V^{2}}\left[(3 \gamma-4)-2(\gamma-1) A v_{1}-\mathcal{S}\right],
\end{aligned}
$$

where

$$
\begin{aligned}
q & =2 \Sigma^{2}+\frac{1}{2} \frac{(3 \gamma-2)+(2-\gamma) V^{2}}{1+(\gamma-1) V^{2}} \Omega \\
\Sigma^{2} & =\Sigma_{+}^{2}+\Sigma_{-}^{2}+\Sigma_{12}^{2}+\Sigma_{13}^{2}+\Sigma_{23}^{2} \\
\mathcal{S} & =\Sigma_{a b} c^{a} c^{b}, \quad c^{a} c_{a}=1, \quad v^{a}=V c^{a}, \\
V^{2} & =v_{1}^{2}+v_{2}^{2}+v_{3}^{2}, \\
T & =\frac{\left[(3 \gamma-4)-2(\gamma-1) A v_{1}\right]\left(1-V^{2}\right)+(2-\gamma) V^{2} \mathcal{S}}{1-(\gamma-1) V^{2}} \\
G_{+} & =1+(\gamma-1) V^{2} .
\end{aligned}
$$


These variables are subject to the constraints

$$
\begin{aligned}
1 & =\Sigma^{2}+A^{2}+N^{2}+\Omega \\
0 & =2 \Sigma_{+} A+2 \Sigma_{-} N+\frac{\gamma \Omega v_{1}}{G_{+}} \\
0 & =-\left[\Sigma_{12}(N+\sqrt{3} A)+\Sigma_{13} \lambda N\right]+\frac{\gamma \Omega v_{2}}{G_{+}} \\
0 & =\left[\Sigma_{13}(N-\sqrt{3} A)+\Sigma_{12} \lambda N\right]+\frac{\gamma \Omega v_{3}}{G_{+}} \\
0 & =A^{2}+3 h\left(1-\lambda^{2}\right) N^{2} .
\end{aligned}
$$

The parameter $\gamma$ will be assumed to be in the interval $\gamma \in(0,2)$. The generalized Friedmann equation, (2.14), yields an expression which effectively defines the energy density $\Omega$. Therefore, the state vector can thus be considered $\mathrm{X}=\left[\Sigma_{+}, \Sigma_{-}, \Sigma_{12}, \Sigma_{13}, \Sigma_{23}, N, \lambda, A, v_{1}, v_{2}, v_{3}\right]$ modulo the constraint equations (equations (2.152.18) ). Thus the dimension of the physical state space is seven (for a given value of the parameter $h$ ). Additional details are presented in 14 .

The dynamical system is invariant under the following discrete symmetries :

$$
\begin{aligned}
\phi_{1}: & {\left[\Sigma_{+}, \Sigma_{-}, \Sigma_{12}, \Sigma_{13}, \Sigma_{23}, N, \lambda, A, v_{1}, v_{2}, v_{3}\right] \mapsto\left[\Sigma_{+}, \Sigma_{-}, \Sigma_{12}, \Sigma_{13}, \Sigma_{23},-N, \lambda,-A,-v_{1},-v_{2},-v_{3}\right] } \\
\phi_{2}: & {\left[\Sigma_{+}, \Sigma_{-}, \Sigma_{12}, \Sigma_{13}, \Sigma_{23}, N, \lambda, A, v_{1}, v_{2}, v_{3}\right] \mapsto\left[\Sigma_{+},-\Sigma_{-}, \Sigma_{13}, \Sigma_{12}, \Sigma_{23},-N, \lambda, A, v_{1}, v_{3}, v_{2}\right] } \\
\phi_{3}: & {\left[\Sigma_{+}, \Sigma_{-}, \Sigma_{12}, \Sigma_{13}, \Sigma_{23}, N, \lambda, A, v_{1}, v_{2}, v_{3}\right] \mapsto\left[\Sigma_{+}, \Sigma_{-}, \mp \Sigma_{12}, \pm \Sigma_{13},-\Sigma_{23}, N,-\lambda, A, v_{1}, \mp v_{2}, \pm v_{3}\right] } \\
\phi_{4}: & {\left[\Sigma_{+}, \Sigma_{-}, \Sigma_{12}, \Sigma_{13}, \Sigma_{23}, N, \lambda, A, v_{1}, v_{2}, v_{3}\right] \mapsto\left[\Sigma_{+}, \Sigma_{-},-\Sigma_{12},-\Sigma_{13}, \Sigma_{23}, N, \lambda, A, v_{1},-v_{2},-v_{3}\right] }
\end{aligned}
$$

These discrete symmetries imply that without loss of generality we can restrict the variables $\lambda \geq 0, A \geq 0$, and $N \geq 0$, since the dynamics in the other regions can be obtained by simply applying one or more of the maps above. The fourth symmetry listed implies that one can add one additional constraint on one of the variables $\Sigma_{12}, \Sigma_{13}, v_{2}$ or $v_{3}$; however, in general there is no natural way to restrict any one of the variables, and hence we will not do so here. Note that any equilibrium point in the region $v_{2}>0$ has a matching equilibrium point in the region $v_{2}<0$.

2.1. Invariant sets. In this analysis we will be concerned with the following invariant sets:

(1) $T\left(V I I_{h}\right)$ : The general tilted type $\mathrm{VII}_{h}$ model. Given by $1<|\lambda|$.

(2) $T(I V)$ : The general tilted type IV model. Given by $\lambda= \pm 1, A>0$.

(3) $T(V)$ : The general tilted type $\mathrm{V}$ model. Given by $N=0, A>0, \lambda= \pm 1$.

(4) $T(I I)$ : The general type II model. Given by $\lambda= \pm 1, A=0$.

(5) $B(I)$ Type I: $N=A=V=0$.

(6) $T_{1}(\mathcal{A})$ : One-component tilted model. Given by $v_{2}=v_{3}=0$.

(7) $\partial T(I)$ "Tilted" vacuum type I: $\Omega=N=A=0$.

We note that the closure of the sets $T(I V)$ and $T\left(V I I_{h}\right)$ are given by

$$
\begin{aligned}
\overline{T\left(V I I_{h}\right)} & =T\left(V I I_{h}\right) \cup T(I I) \cup B(I) \cup \partial T(I), \\
\overline{T(I V)} & =T(I V) \cup T(V) \cup T(I I) \cup B(I) \cup \partial T(I) .
\end{aligned}
$$

Since the boundaries may play an important role in the evolution of the dynamical system we must consider all of the sets in the decomposition (2.19).

\section{Future Asymptotic Behaviour}

3.1. Qualitative Analysis. In order to study the future asymptotic behaviour of tilted type IV and $\mathrm{VII}_{h}$ models, as a first step we will consider all the equilibrium points of the closed sets $\overline{T(I V)}$ and $\overline{T\left(V I I_{h}\right)}$. Fortunately, much work has already been completed in this regard. For instance, the Bianchi tilted type II models were studied in some detail by Hewitt, Bridson and Wainwright [10]. They found that the future 
asymptotic state is either a flat Friedman-Lemaitre model $(0<\gamma \leq 2 / 3)$, a non-tilted Bianchi type II model $(2 / 3<\gamma \leq 10 / 7)$, an intermediately tilted Bianchi type II model $(10 / 7<\gamma<14 / 9)$, a line of tilted points for $\gamma=14 / 9$ or an extremely tilted Bianchi type II model when $14 / 9<\gamma<2$. Similarly, in a paper by Hewitt and Wainwright 9] in which the irrotational Bianchi type V models were analysed, it was found again that the flat Friedman-Lemaitre model is stable for $0<\gamma<2 / 3$, a non-tilted Milne model is stable for $2 / 3<\gamma<4 / 3$ and an intermediately tilted Milne model is stable for $4 / 3<\gamma<2$. Therefore, if we are interested in determining the future asymptotic state of the general tilting Bianchi type IV and $\mathrm{VII}_{h}$ models, we need only determine whether the corresponding equilibrium points are stable in the full Bianchi IV or $\mathrm{VII}_{h}$ phase space. In particular, all equilibrium points in $\partial T(I)$ are Kasner circles and related equilibrium points. None of these equilibrium points are stable into the future and consequently we will not list these below. On the other hand, the set $\partial T(I)$ is essential in the analysis of the past asymptotic behaviour. We should also point out that for the equilibrium points with $N=0$ a different gauge has been used in order to determine their stability.

\subsection{1. $B(I)$ : Equilibrium points of Bianchi type I.}

(1) $\mathcal{I}(I):[0,0,0,0,0,0, \lambda, 0,0,0,0]$ where $\lambda$ is an unphysical parameter. This represents the flat FriedmanLemaitre model.

Eigenvalues:

$$
-\frac{3(2-\gamma)}{2}[\times 5], \frac{3 \gamma-2}{2}[\times 2] .
$$

The remaining equilibrium points are all in $\partial T(I)$.

3.1.2. $T(I I)$ : Equilibrium points of Bianchi type II. All the tilted equilibrium points come in pairs. These represent identical solutions (they differ by a frame rotation); however, since their embeddings in the full state space are inequivalent, two of their eigenvalues are different. All equilibrium points have an unstable direction with eigenvalue $-2 \sqrt{3} \Sigma_{23}$ corresponding to the variable $A$.

(1) $\mathcal{C S}(I I):\left[\frac{2-3 \gamma}{16}, 0,0,0, \frac{(2-3 \gamma) \sqrt{3}}{16}, \frac{\sqrt{3(3 \gamma-2)(2-\gamma)}}{8}, 1,0,0,0,0\right], 2 / 3<\gamma<2$. This is the Collins-Stewart perfect fluid Bianchi II solution.

Eigenvalues:

$$
\frac{3(3 \gamma-2)}{8},-\frac{3}{4}(2-\gamma)\left(1 \pm \sqrt{1-\frac{(6-\gamma)(3 \gamma-2)}{(2-\gamma)}}\right),-\frac{3(2-\gamma)}{2}[\times 2], \frac{3(7 \gamma-10)}{8}[\times 2]
$$

(2) $\mathcal{H}_{1}(I I):\left[\frac{4-3 \gamma}{2},-\frac{\epsilon \sqrt{3}}{4} \sqrt{\frac{(11 \gamma-10)(2-\gamma)(7 \gamma-10)}{17 \gamma-18}}, 0,0,-\frac{\sqrt{3}}{4}(2-\gamma), \frac{\sqrt{3}}{2} \sqrt{\frac{(2-\gamma)(5 \gamma-4)(3 \gamma-4)}{17 \gamma-18}}, 1,0, \epsilon V, 0,0\right], 10 / 7<$ $\gamma<2$. This is Hewitt's tilted Bianchi II model with $\Sigma^{2}=\frac{(3 \gamma-4)\left(9 \gamma^{2}-20 \gamma+12\right)}{17 \gamma-18}, \Omega=\frac{3(2-\gamma)\left(21 \gamma^{2}-24 \gamma+4\right)}{4(17 \gamma-18)}, V^{2}=$ $\frac{(3 \gamma-4)(7 \gamma-10)}{(11 \gamma-10)(5 \gamma-4)}$ and $\epsilon^{2}=1$.

Three of the eigenvalues are $\frac{3(9 \gamma-14)}{4}, \frac{3(2-\gamma)}{2}, \frac{3(7 \gamma-10)}{4}$, with the remaining four eigenvalues being the roots of a nasty equation.

(3) $\mathcal{H}_{2}(I I):\left[\frac{9 \gamma-14}{8}, 0, \frac{\epsilon}{2} \alpha, \frac{\epsilon}{2} \alpha,-\frac{\sqrt{3}}{8}(5 \gamma-6), \frac{\sqrt{3}}{2} \sqrt{\frac{(2-\gamma)(5 \gamma-4)(3 \gamma-4)}{17 \gamma-18}}, 1,0,0, \epsilon \frac{V}{\sqrt{2}},-\epsilon \frac{V}{\sqrt{2}}\right], 10 / 7<\gamma<2$. This is Hewitt's tilted Bianchi II model where $\Sigma^{2}, \Omega$ and $V$ are the same as for $\mathcal{H}_{2}(I I)$, and $\alpha=-\frac{\sqrt{6}}{4} \sqrt{\frac{(11 \gamma-10)(2-\gamma)(7 \gamma-10)}{17 \gamma-18}}$ and $\epsilon^{2}=1$.

Three of the eigenvalues are $\frac{3(9 \gamma-14)}{4}, \frac{3(5 \gamma-6)}{4},-\frac{3(7 \gamma-10)}{4}$, with the remaining four being the roots of the same nasty equation as for $\mathcal{H}_{1}(I I)$.

(4) $\mathcal{L}_{1}(I I): \quad\left[-\frac{1}{3},-\frac{\epsilon \sqrt{57\left(27 \beta^{2}+2\right)\left(64-81 \beta^{2}\right)}}{342}, \frac{1}{2} \beta,-\frac{1}{2} \beta,-\frac{\sqrt{3}}{9}, \frac{\sqrt{19\left(27 \beta^{2}+4\right)\left(17-27 \beta^{2}\right)}}{114}, 1,0, \epsilon V, 0,0\right], \gamma=14 / 9$. This is an intermediately tilted line bifurcation where $0<\beta^{2}<\frac{8}{27}, V^{2}=\frac{3\left(27 \beta^{2}+4\right)\left(27 \beta^{2}+2\right)}{\left(17-27 \beta^{2}\right)\left(64-81 \beta^{2}\right)}$ and 
$\Omega=\frac{1}{684}\left(1458 \beta^{4}-1215 \beta^{2}+472\right)$.

Three of the Eigenvalues are $0, \frac{2}{3}, \frac{2}{3}$.

(5) $\mathcal{L}_{2}(I I):\left[0,-\frac{\beta \sqrt{19\left(64-81 \beta^{2}\right)}}{38}, \frac{\epsilon}{2}(\alpha+\beta), \frac{\epsilon}{2}(\alpha-\beta),-\frac{2 \sqrt{3}}{9}, \frac{\sqrt{19\left(27 \beta^{2}+4\right)\left(17-27 \beta^{2}\right)}}{114}, 1,0, \frac{3 \sqrt{3} \beta V}{\rho}, \frac{\epsilon V}{\rho},-\frac{\epsilon V}{\rho}\right], \gamma=$ 14/9. This is an intermediately tilted line bifurcation where $0<\beta^{2}<\frac{8}{27}, \alpha=\frac{\sqrt{57\left(64-81 \beta^{2}\right)}}{171}, \rho=$ $\sqrt{27 \beta^{2}+2}, V^{2}=\frac{3\left(27 \beta^{2}+4\right)\left(27 \beta^{2}+2\right)}{\left(17-27 \beta^{2}\right)\left(64-81 \beta^{2}\right)}, \Omega=\frac{1}{684}\left(1458 \beta^{4}-1215 \beta^{2}+472\right)$ and $\epsilon^{2}=1$.

Three of the Eigenvalues are $0, \frac{4}{3},-\frac{2}{3}$.

(6) $\mathcal{E}_{1}(I I):\left[-\frac{1}{3},-\frac{2 \epsilon \sqrt{190}}{57}, \frac{\sqrt{6}}{9},-\frac{\sqrt{6}}{9},-\frac{\sqrt{3}}{9}, \frac{\sqrt{57}}{19}, 1,0, \epsilon, 0,0\right], 0<\gamma<2$. This is an extremely tilted Bianchi II, with $\Omega=20 / 57, \Sigma^{2}=28 / 57$ and $\epsilon^{2}=\delta^{2}=1$.

Eigenvalues:

$$
\frac{-2(9 \gamma-14)}{3(2-\gamma)}, 2 / 3,2 / 3, \frac{-1}{3} \pm \frac{1}{57} \sqrt{-20615 \pm 456 \sqrt{311}}
$$

(7) $\mathcal{E}_{2}(I I):\left[0,-\frac{4 \epsilon \sqrt{285}}{171},-\frac{\delta \sqrt{6}}{9}\left(1+\frac{\epsilon \sqrt{95}}{19}\right),-\frac{\delta \sqrt{6}}{9}\left(1-\frac{\epsilon \sqrt{95}}{19}\right),-\frac{2 \sqrt{3}}{9}, \frac{\sqrt{57}}{19}, 1,0, \frac{2 \epsilon \sqrt{5}}{5},-\epsilon \delta \frac{\sqrt{10}}{10}, \epsilon \delta \frac{\sqrt{10}}{10}\right], 0<$ $\gamma<2$. This is an extremely tilted Bianchi II, with $\Omega=20 / 57, \Sigma^{2}=28 / 57$ and $\epsilon^{2}=\delta^{2}=1$.

Eigenvalues:

$$
\frac{-2(9 \gamma-14)}{3(2-\gamma)}, 4 / 3,-2 / 3, \frac{-1}{3} \pm \frac{1}{57} \sqrt{-20615 \pm 456 \sqrt{311}}
$$

3.1.3. $T(V)$ : Equilibrium points of Bianchi $V$.

(1) $\mathcal{I}(V):[0,0,0,0,0,0,1, A, 0,0,0], 0<A<1, \gamma=2 / 3$. Represents a FRW model with a $\gamma=2 / 3$ fluid.

Eigenvalues:

$$
0[\times 2],-2[\times 5] .
$$

(2) $\mathcal{M}(V):[0,0,0,0,0,0,1,1,0,0,0], 0<\gamma<2$. This represents the Milne universe. $\left(\Sigma^{2}=0, \Omega=\right.$ $\left.0, V^{2}=0\right)$

Eigenvalues:

$$
0,-2[\times 2],-(3 \gamma-2),(3 \gamma-4)[\times 3] .
$$

The zero eigenvalue arises due to the fact that this is part of the one-parameter family of plane-wave solutions.

(3) $\widetilde{\mathcal{M}}(V):\left[0,0,0,0,0,0,1,1, \frac{3 \gamma-4}{2(\gamma-1)}, 0,0\right], 6 / 5<\gamma<2$. This represents an "intermediately tilted" Milne model $\left(\Sigma^{2}=0, \Omega=0, V^{2}=\frac{(3 \gamma-4)^{2}}{4(\gamma-1)^{2}}\right)$.

Eigenvalues:

$$
0,-2[\times 2], \frac{(3 \gamma-4)}{2(\gamma-1)}[\times 2],-\frac{(3 \gamma-4)(5 \gamma-6)}{(9 \gamma-10)(\gamma-1)}
$$

Again the zero eigenvalue is associated with there being a non-isolated line of equilibria.

(4) $\mathcal{M}_{ \pm}(V):[0,0,0,0,0,0,1,1, \pm 1,0,0], 0<\gamma<2$. This represents "extremely tilted" Milne models $\left(\Sigma^{2}=0, \Omega=0, V^{2}=1\right)$.

Eigenvalues:

$$
\mathcal{M}_{+}: 0[\times 2],-2[\times 2], 1[\times 2], 2, \quad \mathcal{M}_{-}: 0,-2[\times 2],-4, \frac{2(5 \gamma-6)}{2-\gamma},-1[\times 2] .
$$

(5) $\mathcal{R}(V):\left[\Sigma_{+}, 0,0,0,0,0,1,\left(1+\Sigma_{+}\right), 1,0,0\right],-1<\Sigma_{+}<0,0<\gamma<2$. This represents a type $\mathrm{V}$ model with a null fluid. 
Eigenvalues:

$$
0[\times 2],-2\left(1+\Sigma_{+}\right)[\times 2],\left(1-2 \Sigma_{+}\right)[\times 2], 2\left(1-2 \Sigma_{+}\right) .
$$

The two zero eigenvalues are associated with this being part of a two-parameter family of equilibrium points $(\mathcal{R}(I V)$ see below $)$.

3.1.4. $T(I V)$ : Equilibrium points of Bianchi type $I V$.

(1) $\mathcal{L}(I V):\left[\Sigma_{+}, \sqrt{-\Sigma_{+}\left(1+\Sigma_{+}\right)}, 0,0,0, \sqrt{-\Sigma_{+}\left(1+\Sigma_{+}\right)}, 1,\left(1+\Sigma_{+}\right), 0,0,0\right],-1<\Sigma_{+}<0,0<\gamma<2$. These represent 'non-tilted' vacuum plane waves.

Eigenvalues:

$$
0,-2\left[\left(1+\Sigma_{+}\right) \pm 2 i \sqrt{3} N\right],-4 \Sigma_{+}-(3 \gamma-2),(3 \gamma-4)+2 \Sigma_{+},(3 \gamma-4)-\Sigma_{+}
$$

(2) $\widetilde{\mathcal{L}}_{ \pm}(I V):\left[\Sigma_{+}, \sqrt{-\Sigma_{+}\left(1+\Sigma_{+}\right)}, 0,0,0, \sqrt{-\Sigma_{+}\left(1+\Sigma_{+}\right)}, 1,\left(1+\Sigma_{+}\right), \pm 1,0,0\right],-1<\Sigma_{+}<0,0<\gamma<2$ These represent 'extremely tilted' vacuum plane waves.

Eigenvalues:

$$
\begin{aligned}
& \widetilde{\mathcal{L}}_{+}: 0,-2\left[\left(1+\Sigma_{+}\right) \pm 2 i \sqrt{3} N\right], 0,2\left(1-2 \Sigma_{+}\right),\left(1-2 \Sigma_{+}\right)[\times 2] . \\
& \widetilde{\mathcal{L}}_{-}: 0,-2\left[\left(1+\Sigma_{+}\right) \pm 2 i \sqrt{3} N\right],-4\left(1+\Sigma_{+}\right),-\frac{2\left(5 \gamma-6+2 \gamma \Sigma_{+}\right)}{2-\gamma},-\left(1+4 \Sigma_{+}\right)[\times 2] .
\end{aligned}
$$

(3) $\widetilde{\mathcal{L}}(I V):\left[\Sigma_{+}, \sqrt{-\Sigma_{+}\left(1+\Sigma_{+}\right)}, 0,0,0, \sqrt{-\Sigma_{+}\left(1+\Sigma_{+}\right)}, 1,\left(1+\Sigma_{+}\right), \frac{3 \gamma-4+2 \Sigma_{+}}{2(\gamma-1)\left(\Sigma_{+}+1\right)}, 0,0\right],-1<\Sigma_{+}<0$, $6 /\left(5+2 \Sigma_{+}\right)<\gamma<2$. These represent 'intermediately tilted' vacuum plane waves.

Eigenvalues:

$$
\begin{aligned}
& 0,-2\left[\left(1+\Sigma_{+}\right) \pm 2 i \sqrt{3} N\right],-\frac{2-\gamma}{\gamma-1}\left(1-2 \Sigma_{+}\right) \\
& -\frac{\left(1-2 \Sigma_{+}\right)\left(5 \gamma-6+2 \gamma \Sigma_{+}\right)\left(3 \gamma-4+2 \Sigma_{+}\right)}{(\gamma-1)\left(9 \gamma-10+4 \Sigma_{+}\left(1-\Sigma_{+}\right)\right)}, \frac{\left(1-2 \Sigma_{+}\right)(3 \gamma-4)}{2(\gamma-1)}[\times 2] .
\end{aligned}
$$

(4) $\widetilde{\mathcal{F}}_{ \pm}(I V):\left[\Sigma_{+}, \sqrt{-\Sigma_{+}\left(1+\Sigma_{+}\right)}, 0,0,0, \sqrt{-\Sigma_{+}\left(1+\Sigma_{+}\right)}, 1,\left(1+\Sigma_{+}\right),-\frac{4-3 \gamma-\Sigma_{+}}{(3-2 \gamma-1)\left(\Sigma_{+}+1\right)}, v_{2},-v_{2}\right]$ where $v_{2}= \pm \frac{\sqrt{2(3 \gamma-4)\left(-1+2 \Sigma_{+}\right)\left(3 \gamma-4-\Sigma_{+}\right)}}{2(3-2 \gamma)\left(1+\Sigma_{+}\right)},-1<\Sigma_{+}<0,\left(4+\Sigma_{+}\right) / 3<\gamma<\min \left[4 / 3,3 /\left(2-\Sigma_{+}\right)\right]$. These represent 'intermediately tilted' vacuum plane waves.

Eigenvalues:

$$
0,-2\left[\left(1+\Sigma_{+}\right) \pm 2 i \sqrt{3} N\right],-\frac{\left(1-2 \Sigma_{+}\right)(5 \gamma-6)}{3-2 \gamma}, 0, \lambda_{6}, \lambda_{7},
$$

where

$$
\begin{aligned}
& \lambda_{6} \lambda_{7}=-\frac{\left(1-2 \Sigma_{+}\right)^{2}(4-3 \gamma)\left(3 \gamma-4-\Sigma_{+}\right)\left(3-2 \gamma+\gamma \Sigma_{+}\right)}{(3-2 \gamma) G\left(\gamma, \Sigma_{+}\right)} \\
& \lambda_{6}+\lambda_{7}=\frac{\left(1-2 \Sigma_{+}\right) F\left(\gamma, \Sigma_{+}\right)}{4(3-2 \gamma)\left(17 \gamma^{2}-40 \gamma+24\right) G\left(\gamma, \Sigma_{+}\right)}
\end{aligned}
$$

and

$$
\begin{aligned}
G\left(\gamma, \Sigma_{+}\right) \equiv & (5 \gamma-6) \Sigma_{+}^{2}-\left(18-25 \gamma+9 \gamma^{2}\right) \Sigma_{+}-3+2 \gamma \\
F\left(\gamma, \Sigma_{+}\right) \equiv & {\left[2\left(17 \gamma^{2}-40 \gamma+24\right) \Sigma_{+}-33 \gamma^{3}+121 \gamma^{2}-152 \gamma+66\right]^{2} } \\
& -9(\gamma-1)^{2}\left(121 \gamma^{4}-736 \gamma^{3}+1664 \gamma^{2}-1656 \gamma+612\right)
\end{aligned}
$$

Here is $G\left(\gamma, \Sigma_{+}\right)<0$ in the whole region under consideration. $F\left(\gamma, \Sigma_{+}\right)=0$, defines a line from $(4 / 3,0)$ to $(4 / 3,-1 / 4)$. 
(5) $\widetilde{\mathcal{E}}_{ \pm}(I V):\left[\Sigma_{+}, \sqrt{-\Sigma_{+}\left(1+\Sigma_{+}\right)}, 0,0,0, \sqrt{-\Sigma_{+}\left(1+\Sigma_{+}\right)}, 1,\left(1+\Sigma_{+}\right), \frac{1+\Sigma_{+}}{3 \Sigma_{+}}, v_{2},-v_{2}\right]$, where $v_{2}= \pm \frac{\sqrt{2\left(4 \Sigma_{+}+1\right)\left(2 \Sigma_{+}-1\right)}}{6 \Sigma_{+}},-1<\Sigma_{+}<-1 / 4,0<\gamma<2$. These represent 'extremely tilted' vacuum plane waves.

Eigenvalues:

$0,-2\left[\left(1+\Sigma_{+}\right) \pm 2 i \sqrt{3} N\right], \frac{\left(1-2 \Sigma_{+}\right)\left(1+2 \Sigma_{+}\right)}{\Sigma_{+}}, 0,-\frac{\left(1-2 \Sigma_{+}\right)\left(1+4 \Sigma_{+}\right)}{3 \Sigma_{+}}, \frac{2\left(1-2 \Sigma_{+}\right)\left(2 \gamma-3-\gamma \Sigma_{+}\right)}{3 \Sigma_{+}(2-\gamma)}$,

(6) $\mathcal{R}(I V):\left[\Sigma_{+},(1-\ell) \sqrt{-\Sigma_{+}\left(1+\Sigma_{+}\right)}, 0,0,0,(1-\ell) \sqrt{-\Sigma_{+}\left(1+\Sigma_{+}\right)}, 1,\left(1+\Sigma_{+}\right), 1,0,0\right],-1<\Sigma_{+}<0$, $0<\ell<1,0<\gamma<2$. These represent non-vacuum plane-waves with a null fluid.

Eigenvalues: Same as for $\widetilde{\mathcal{L}}_{+}(I V)$.

3.1.5. $T\left(V I I_{h}\right)$ : Equilibrium points of Bianchi type $V I I_{h}$. For all of the vacuum equilibrium points the group parameter, $h$, is given by

and we have defined $\tilde{h} \equiv 1 / \sqrt{h}$.

$$
3 h \Sigma_{+}\left(1-\lambda^{2}\right)=\left(1+\Sigma_{+}\right)
$$

(1) $\mathcal{L}\left(V I I_{h}\right):\left[\Sigma_{+}, \sqrt{-\Sigma_{+}\left(1+\Sigma_{+}\right)}, 0,0,0, \sqrt{-\Sigma_{+}\left(1+\Sigma_{+}\right)}, \lambda,\left(1+\Sigma_{+}\right), 0,0,0\right],-1<\Sigma_{+}<0,1<\lambda$, $0<\gamma<2$. These represent 'non-tilted' vacuum plane waves.

Eigenvalues:

$0,-2\left[\left(1+\Sigma_{+}\right) \pm 2 i \sqrt{3} N\right],-4 \Sigma_{+}-(3 \gamma-2),(3 \gamma-4)+2 \Sigma_{+},(3 \gamma-4)-\Sigma_{+} \pm i \tilde{h}\left(1+\Sigma_{+}\right)$.

(2) $\widetilde{\mathcal{L}}_{ \pm}\left(V I I_{h}\right):\left[\Sigma_{+}, \sqrt{-\Sigma_{+}\left(1+\Sigma_{+}\right)}, 0,0,0, \sqrt{-\Sigma_{+}\left(1+\Sigma_{+}\right)}, \lambda,\left(1+\Sigma_{+}\right), \pm 1,0,0\right],-1<\Sigma_{+}<0,1<\lambda$ $0<\gamma<2$. These represent 'extremely tilted' vacuum plane waves.

Eigenvalues:

$$
\begin{aligned}
& \widetilde{\mathcal{L}}_{+}: \quad 0,-2\left[\left(1+\Sigma_{+}\right) \pm 2 i \sqrt{3} N\right], 0,2\left(1-2 \Sigma_{+}\right),\left(1-2 \Sigma_{+}\right)[\times 2] \\
& \widetilde{\mathcal{L}}_{-}: 0,-2\left[\left(1+\Sigma_{+}\right) \pm 2 i \sqrt{3} N\right],-4\left(1+\Sigma_{+}\right),-\frac{2\left(5 \gamma-6+2 \gamma \Sigma_{+}\right)}{2-\gamma},-\left(1+4 \Sigma_{+}\right) \pm 2 i \tilde{h}\left(1+\Sigma_{+}\right) .
\end{aligned}
$$

(3) $\tilde{\mathcal{L}}\left(V I I_{h}\right):\left[\Sigma_{+}, \sqrt{-\Sigma_{+}\left(1+\Sigma_{+}\right)}, 0,0,0, \sqrt{-\Sigma_{+}\left(1+\Sigma_{+}\right)}, \lambda,\left(1+\Sigma_{+}\right), \frac{3 \gamma-4+2 \Sigma_{+}}{2(\gamma-1)\left(\Sigma_{+}+1\right)}, 0,0\right],-1<\Sigma_{+}<$ $0,1<\lambda, 6 /\left(5+2 \Sigma_{+}\right)<\gamma<2$. These represent 'intermediately tilted' vacuum plane waves.

Eigenvalues:

$$
\begin{aligned}
& 0,-2\left[\left(1+\Sigma_{+}\right) \pm 2 i \sqrt{3} N\right],-\frac{2-\gamma}{\gamma-1}\left(1-2 \Sigma_{+}\right), \\
& -\frac{\left(1-2 \Sigma_{+}\right)\left(5 \gamma-6+2 \gamma \Sigma_{+}\right)\left(3 \gamma-4+2 \Sigma_{+}\right)}{(\gamma-1)\left(9 \gamma-10+4 \Sigma_{+}\left(1-\Sigma_{+}\right)\right)}, \frac{\left(1-2 \Sigma_{+}\right)(3 \gamma-4)}{2(\gamma-1)} \pm i \tilde{h}\left(1-v_{1}\right)\left(1+\Sigma_{+}\right) .
\end{aligned}
$$

(4) $\mathcal{R}\left(V I I_{h}\right):\left[\Sigma_{+},(1-\ell) \sqrt{-\Sigma_{+}\left(1+\Sigma_{+}\right)}, 0,0,0,(1-\ell) \sqrt{-\Sigma_{+}\left(1+\Sigma_{+}\right)}, \lambda,\left(1+\Sigma_{+}\right), 1,0,0\right],-1<\Sigma_{+}<$ $0,0<\ell<1,1<\lambda, 0<\gamma<2$. Non-vacuum plane-wave with a null fluid. The group parameter is given by $3 h \Sigma_{+}\left(1-\lambda^{2}\right)(1-\ell)=\left(1+\Sigma_{+}\right)$.

Eigenvalues: Same as for $\widetilde{\mathcal{L}}_{+}\left(V I I_{h}\right)$.

3.2. Local stability of equilibrium points. We quickly observe that none of the equilibria in the Bianchi type I and Bianchi type II invariant sets are late-time attractors for the Bianchi IV or $\mathrm{VII}_{h}$ models when $2 / 3<\gamma<2$. For the Bianchi type $\mathrm{V}$ model, only the isotropic Milne universes can act as future attractors. However, as can be seen, these equilibrium points are the isotropic limits of the plane-wave equilibrium points of Bianchi type IV and can be extracted directly from this analysis. Furthermore, the isotropic limit of the type $\mathrm{VII}_{h}$ model can also be directly extracted from the type $\mathrm{VII}_{h}$ analysis (even though $\lambda$ diverges here). 
Figure 1. Stability of the Plane-Wave Vacuum Solutions. The regions where the different plane-wave equilibrium points are attractors for the Bianchi type IV with $v_{2}+v_{3} \geq 0$. In the left figure, all the boundaries along the left edge marks the instability of the energy density, $\Omega$. The right figure is a magnified region of the left one showing the loophole (figure taken from 14).
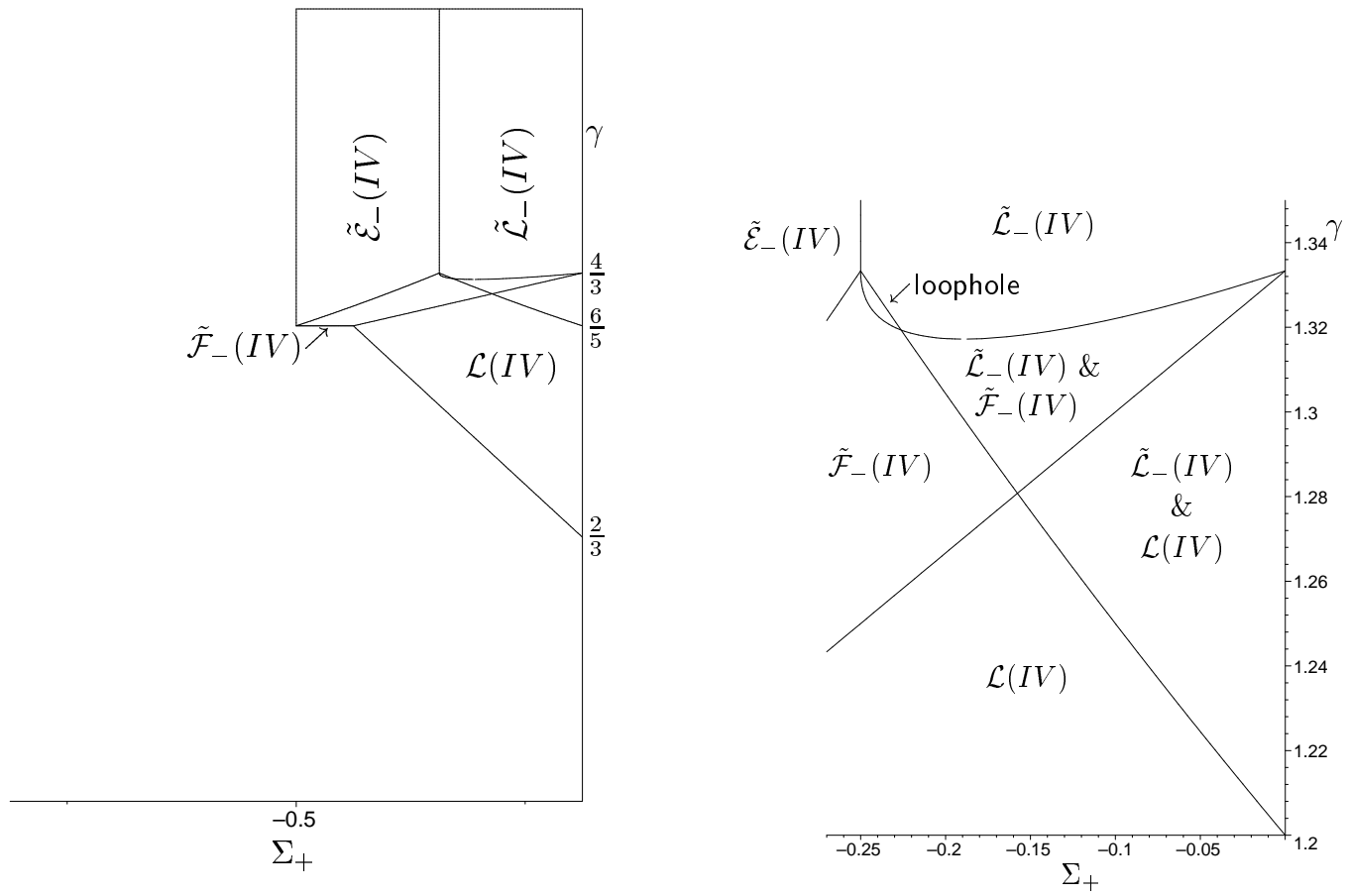

The stability analysis of the type IV model can be summarized as follows: For $2 / 3<\gamma<2$, the only equilibrium points that are future attractors in $\overline{T(I V)}$ are:

(1) $\mathcal{L}(I V)$ : stable for $\frac{2-4 \Sigma_{+}}{3}<\gamma<\frac{4+\Sigma_{+}}{3}$.

(2) $\tilde{\mathcal{L}}_{-}(I V)$ : stable $\frac{6}{5+2 \Sigma_{+}}<\gamma<2,-\frac{1}{4}<\Sigma_{+}<0$.

(3) $\tilde{\mathcal{F}}_{ \pm}(I V): \tilde{\mathcal{F}}_{-}(I V)$ stable in $\left(v_{2}+v_{3}\right) \geq 0{ }^{1}$ for $\max \left(\frac{6}{5}, \frac{4+\Sigma_{+}}{3}\right)<\gamma<\min \left(\gamma_{0}, \frac{3}{2-\Sigma_{+}}\right)$

(4) $\tilde{\mathcal{E}}_{ \pm}(I V): \tilde{\mathcal{E}}_{-}(I V)$ stable for $-\frac{1}{2}<\Sigma_{+}<-\frac{1}{4}, \frac{3}{2-\Sigma_{+}}<\gamma<2$.

Here, we have defined $\gamma_{0}$ for any given value of $\Sigma_{+}$as $F\left(\gamma_{0}, \Sigma_{+}\right)=0$, where $F\left(\gamma, \Sigma_{+}\right)$is defined in eq.(3.1). Note that there is a region where there are two co-existing future attractors; namely, the region where $\frac{6}{5+2 \Sigma_{+}}<\gamma<\gamma_{0}$. Also, there is a tiny region $\gamma_{0}<\gamma<\frac{6}{5+2 \Sigma_{+}}$(from now on called the 'loophole') which does not contain any stable equilibrium points (see Figure 1).

The stability analysis of the equilibrium points of type $\mathrm{VII}_{h}$ can be summarized as follows: For $2 / 3<$ $\gamma<2$, the only equilibrium points that are future attractors in $\overline{T\left(V I I_{h}\right)}$ are:

(1) $\mathcal{L}\left(V I I_{h}\right)$ : stable for $\frac{2-4 \Sigma_{+}}{3}<\gamma<\frac{4+\Sigma_{+}}{3}$.

(2) $\tilde{\mathcal{L}}_{-}\left(V I I_{h}\right):$ stable for $\frac{6}{5+2 \Sigma_{+}}<\gamma<2,-\frac{1}{4}<\Sigma_{+}<0$.

\footnotetext{
${ }^{1} \tilde{\mathcal{F}}_{+}(I V)$ is stable in the same region of $\gamma$ for the half $\left(v_{2}+v_{3}\right) \leq 0$.
} 
Figure 2. The figure below displays the dynamical behaviour for values of the parameters near the loophole for Bianchi type IV non-vacuum tilted models. The value of $\gamma$ is 1.325 and the initial conditions are chosen to be close to the plane wave solutions for illustrative purposes only. A cross indicates the locations of $\widetilde{\mathcal{E}}_{-}(I V)$, diamonds indicate the position of $\widetilde{\mathcal{L}}_{-}(I V)$ while an asterisk indicates the initial condition. The equilibrium point $\widetilde{\mathcal{F}}_{-}(I V)$ is located in the center of the spiral and the center of the closed loop. Note how the dynamical behaviour depends on the final value of $\Sigma_{+}$.

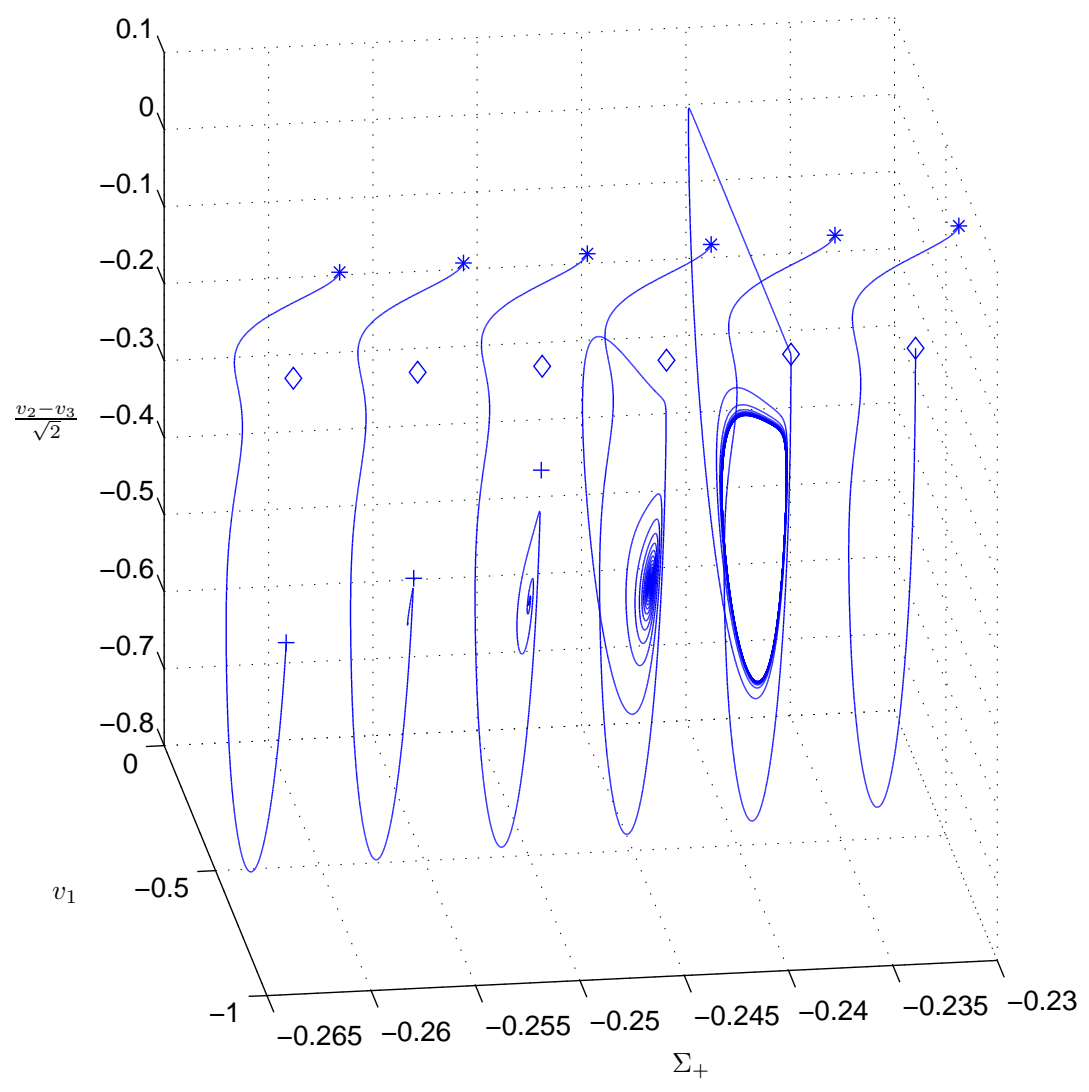

3.3. The Bianchi type IV loophole. As pointed out in 14 , in the limiting vacuum case (i.e., lim $\Omega \rightarrow 0)$, the stability of the plane wave attractors changed as a function of both the parameter $\gamma$ and the terminal value of $\Sigma_{+}$, henceforth denoted as $\Sigma_{+}^{*}$. As we have seen from the above there also exists a region of parameter space (here we consider the terminal value $\Sigma_{+}^{*}$ as a parameter) which does not contain any stable planewave equilibrium points (the loophole). With the use of the bifurcation diagram and through subsequent numerical experimentation, a limit cycle was found for values of $\gamma$ and $\Sigma_{+}^{*}$ inside the loophole, see Figure 1 This limit cycle acts as an attractor and will be referred to as the Mussel attractor (see section 5 and Figure 2 in [14]). Figures 2 and 3 indicate how the dynamical behaviour of the Mussel attractor changes for $\gamma=1.325$ and different limiting values of $\Sigma_{+}$.

We note that the limit cycles observed in Figure 2 above are actually a continuum of limit cycles side by side. The limiting value of $\Sigma_{+}$is the determining factor in the final asymptotic state of the system. Of course one might argue that the likelihood of actually falling into this family of limit cycles is small. Again 
FiguRE 3. The figure below displays the dynamical behaviour for values of the parameters near the loophole for Bianchi type IV non-vacuum tilted models. A cross indicates the locations of $\widetilde{\mathcal{E}}_{-}(I V)$, diamonds indicate the position of $\widetilde{\mathcal{L}}_{-}(I V)$ while an asterisk indicates the initial condition. The equilibrium point $\widetilde{\mathcal{F}}_{-}(I V)$ is located in the center of the spiral and the center of the closed loop. Note how the dynamical behaviour depends on the value of $\Sigma_{+}^{*}$. Each trajectory represents the vacuum limit of the orbits observed in the figure above.

The circular arc represents the boundary of the phase space.
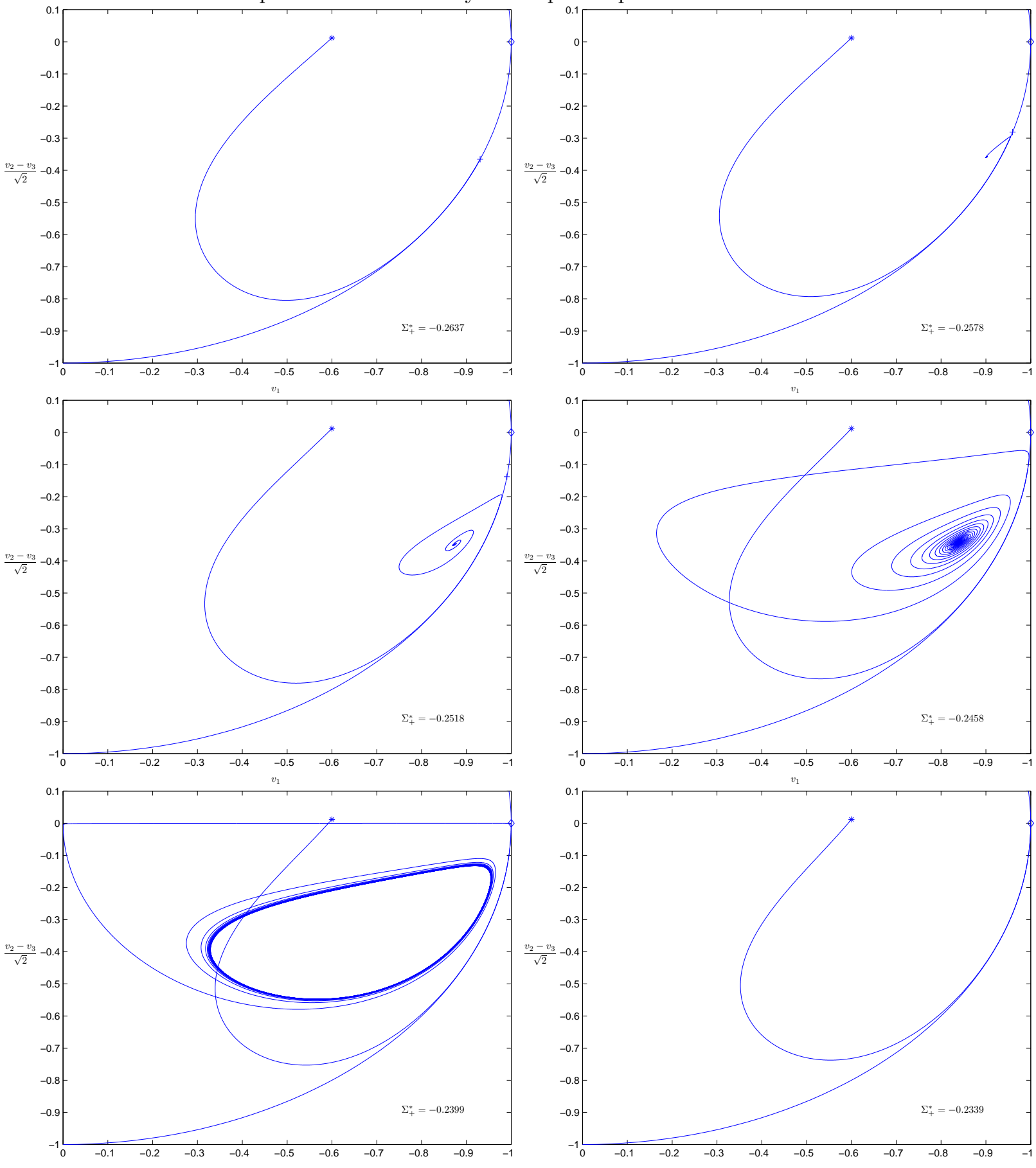
through some numerical experimentation it can be shown that there is a set of Bianchi IV initial conditions far from the plane-wave solutions of non-zero measure that have these limit cycles as future attractors for

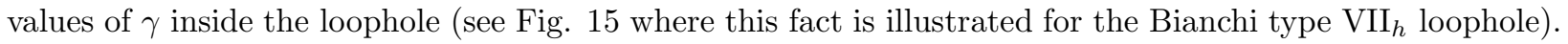

3.4. Behaviour of the System in General. One of the most important points and argument in this and the companion paper is that at late times $\Omega \rightarrow 0$ and the variables $\Sigma_{a b}, N$, etc., 'freeze' into a particular asymptotic value. We have not been able to prove this rigorously, however, there is much evidence to support the following:

Conjecture 3.1. Except for a set of measure zero, non-inflationary $(2 / 3<\gamma<2)$ tilted perfect fluid Bianchi type $I V$ and $V I I_{h}$ models will at late times approach a vacuum state; i.e.

$$
\lim _{\tau \rightarrow \infty} \Omega=0 .
$$

There are several results that support this. First, a local stability analysis shows that there are always stable vacuum solutions. So the conjecture is at least valid locally. Second, we know that there are no non-vacuum equilibrium points acting as local attractors; the preceding analysis excluded this. Third, in our fairly extensive numerical analysis no evidence for any other behaviour has been found. In particular, Figure 2 shows how the variable $\Sigma_{+}$freezes in to a particular value of $\Sigma_{+}$. In Appendix $\$$ more examples of this behaviour are shown and we can see explicitly how $\Omega \rightarrow 0$ and $\Sigma_{+} \rightarrow \Sigma_{+}^{*}$.

The local analysis also shows that the variable $\Sigma_{a b}, N$, etc. 'freeze in' to particular values $\Sigma_{a b}^{*}, N^{*}$. After this freezing has occurred, the system of equations effectively decouples and the equations governing the tilt velocities separate. In effect, the tilt equations reduce to the much simpler form $v_{i}=F\left(v_{j} ; \Sigma_{+}^{*}, \Sigma_{-}^{*}, \cdots\right)$, and hence, we can, at late times, treat all the variables, except $v_{1}, v_{2}$ and $v_{3}$, as constants. This 'freezing in' process makes these models amenable to analytical investigations, which was done in [14; the analysis provides numerical justification for this freezing process (e.g., see Figure 21). In fact, the numerical investigations show that the time scale of this freezing process is typically much shorter than many of the other features present; e.g. the oscillation in the type IV loophole (see Appendix A]. In the Appendix we have included a sample of numerical integrations. For the plots we have chosen the particularly interesting values $\gamma=1$ (dust), 1.325 and $4 / 3$ (radiation). In all of the plots we can see how quickly the energy-density, $\Omega$, tends to zero and the variable $\Sigma_{+}$freezes in. On the other hand, the velocities typically take longer to settle into a particular asymptotic state (as explicit examples of this, see Figures 11] and 12). We should point out that the numerical integrations are done in the full 7-dimensional state space; however, for some figures the reduced system is used for illustrative purposes only.

\section{Dealing with Closed Loops}

The dynamical system contains closed periodic orbits. Some of these closed curves act as limit cycles and take the role as attractors for the dynamical system. However, closed loops are difficult to find and describe in full generality.

In our case, the 'freezing in' process enables us to consider the reduced system of equations describing the behaviour of the tilt velocities in a vacuum plane-wave background, see [14]. We utilize the identity

$$
\left(v_{2}^{2}+v_{3}^{2}\right)^{2}=\left(2 v_{2} v_{3}\right)^{2}+\left(v_{2}^{2}-v_{3}^{2}\right)^{2},
$$

and define $(x, \rho, \theta)$ by

$$
x=v_{1}, \quad \rho \equiv v_{2}^{2}+v_{3}^{2}, \quad 2 v_{2} v_{3}=\rho \sin \theta, \quad\left(v_{2}^{2}-v_{3}^{2}\right)=\rho \cos \theta .
$$


Then we have for the reduced system

$$
\begin{aligned}
x^{\prime} & =\left(T+2 \Sigma_{+}^{*}\right) x-\left(A^{*}+\sqrt{3} N^{*} \cos \theta\right) \rho, \\
\rho^{\prime} & =2\left(T-\Sigma_{+}^{*}+A^{*} x-\alpha \cos \theta\right) \rho, \\
\theta^{\prime} & =2 \alpha\left(\lambda^{*}+\sin \theta\right),
\end{aligned}
$$

where $\alpha=\sqrt{3}(1-x) N^{*}$ and, by use of the discrete symmetry $\phi_{4}$, we can assume that $0 \leq \theta<2 \pi$. Furthermore, these variables are bounded by

$$
0 \leq \rho, \quad V^{2}=x^{2}+\rho \leq 1
$$

An asterisk has been added to the variables to emphasize that these should be thought of as the limit values for the full system.

We will utilize the following fact: Assume that $c(\tau)$ is a (solution) curve, then if $f$ is a function in the state space,

$$
\int_{t_{1}}^{t_{2}} f^{\prime}(c(\tau)) d \tau=f\left(c\left(t_{2}\right)\right)-f\left(c\left(t_{1}\right)\right)
$$

In particular, if $c(\tau)$ is a closed periodic orbit,

$$
\oint f^{\prime}(c(\tau)) d \tau=0
$$

For a periodic orbit, $c$, with period $T_{n}$, we introduce the average of a variable $B$ :

$$
\langle B\rangle \equiv \frac{1}{T_{n}} \oint_{c} B d \tau
$$

We can also say something about the stability of a closed periodic orbit. For example, consider the evolution equation for $\Omega$, which we write as $\Omega^{\prime}=\Omega \lambda_{\Omega}$. Assume that $c(\tau)$ is a closed periodic orbit with period $T_{n}$. Then for every $\tau_{0}$ and $\epsilon>0$, there exists a solution curve, $\tilde{c}(\tau)=c(\tau)+\delta(\tau)$, for which

$$
|\delta(\tau)|<\epsilon, \quad \tau_{0}<\tau<\tau_{0}+T_{n} .
$$

(This follows from Proposition 4.2, page 104, in 24.) Hence, the curve $\tilde{c}(\tau)$ can be used to approximate the closed curve $c(\tau)$. Using $\tilde{c}(\tau)$ we get

$$
\ln \left[\frac{\Omega\left(\tau_{0}+T_{n}\right)}{\Omega\left(\tau_{0}\right)}\right]=\int_{\tau_{0}}^{\tau_{0}+T_{n}} \frac{\Omega^{\prime}}{\Omega} d \tau=\int_{\tau_{0}}^{\tau_{0}+T_{n}} \lambda_{\Omega} d \tau=T_{n}\left\langle\lambda_{\Omega}\right\rangle+\mathcal{O}(\epsilon) .
$$

Since $\epsilon$ can be arbitrarily small, we can approximate

$$
\Omega\left(\tau_{0}+T_{n}\right) \approx \Omega\left(\tau_{0}\right) \exp \left(T_{n}\left\langle\lambda_{\Omega}\right\rangle\right) .
$$

Hence, if $\left\langle\lambda_{\Omega}\right\rangle<0$, then $\Omega\left(\tau_{0}+T_{n}\right)<\Omega\left(\tau_{0}\right)$ for a sufficiently small perturbation; i.e., the closed curve is stable with respect to $\Omega$. If, on the other hand, $\left\langle\lambda_{\Omega}\right\rangle>0$ then the closed curve is unstable with respect to a perturbation of $\Omega$. This result is the corresponding local stability criterion for periodic orbits.

By calculating $\langle 1-x\rangle$ (in two different ways) we are able to find an expression for $T_{n}$ for orbits in the type $\mathrm{VII}_{h}$ model:

$$
T_{n}=\frac{n \pi \sqrt{h}}{\left(1+\Sigma_{+}^{*}\right)(1-\langle x\rangle)},
$$

where $n$ is an integer. This expression involves the group parameter $h$, and we note that a larger $h$ implies longer orbital period.

We also need, 
Lemma 4.1. Assume that there is a closed properly ${ }^{2}$ periodic orbit, $c(\tau)$, for the dynamical system (4.2). Then

$$
\left\langle\frac{(1-x)\left(v_{2}^{2}-v_{3}^{2}\right)}{v_{2}^{2}+v_{3}^{2}}\right\rangle=0
$$

Proof. For $\left(\lambda^{*}\right)^{2}>1, \theta$ is a strictly monotonic function, so in this case we can use $\theta$ as a variable along the curve. Furthermore, $\theta\left(\tau_{0}+T_{n}\right)=\theta_{0}+2 \pi n$, where $n \in \mathbb{Z}$, which gives

$$
\oint 2 \alpha \cos \theta d \tau=\int_{\theta_{0}}^{\theta_{0}+2 \pi n} \frac{\cos \theta}{\lambda^{*}+\sin \theta} d \theta=\left.\ln \left(\lambda^{*}+\sin \theta\right)\right|_{\theta_{0}} ^{\theta_{0}+2 \pi n}=0 .
$$

Since $\alpha=\sqrt{3} N^{*}(1-x)$, the Lemma now follows for $\left(\lambda^{*}\right)^{2}>1$. For $\lambda^{*}=1$ the equation for $\theta$ implies that the periodic curve must be in the invariant set $\sin \theta=-1$. Thus the Lemma is trivially satisfied in that case.

Theorem 4.2. Assume that there is a closed properly periodic orbit, $c(\tau)$, for the dynamical system 4.2). Then either

$$
\mathcal{C}: \quad\langle x\rangle=-\frac{(3 \gamma-4)-\Sigma_{+}^{*}}{\left(1+\Sigma_{+}^{*}\right)(3-2 \gamma)}, \quad\left\langle\lambda_{\Omega}\right\rangle=-\frac{\left(1-2 \Sigma_{+}^{*}\right)(5 \gamma-6)}{3-2 \gamma}
$$

or $V=1$ and

$$
\mathcal{E}: \quad\langle x\rangle=\frac{1+\Sigma_{+}^{*}}{3 \Sigma_{+}^{*}}, \quad\left\langle\lambda_{\Omega}\right\rangle=\frac{\left(1-2 \Sigma_{+}^{*}\right)\left(1+2 \Sigma_{+}^{*}\right)}{\Sigma_{+}^{*}} .
$$

Proof. Assume first that there exists a closed periodic orbit with $V<1$ and $\rho>0$. Then, using the evolution equation for $V$, we get

$$
(3 \gamma-4)\langle f(V)\rangle-2(\gamma-1) A^{*}\langle x f(V)\rangle-\langle f(V) \mathcal{S}\rangle=0,
$$

for any analytic function $f(V)$ on $(0,1)$. Using Lemma 4.1 the equation for $\rho^{\prime}$ yields

$$
\langle T\rangle-\Sigma_{+}^{*}+A^{*}\langle x\rangle=0 .
$$

Using eq. 4.15) with $f(V)=V^{2} / G_{-}$we obtain $\langle T\rangle=\langle\mathcal{S}\rangle$. Using eq. (4.15) with $f(V)=1$ we can solve for $\langle x\rangle$, which yields

$$
\langle x\rangle=-\frac{(3 \gamma-4)-\Sigma_{+}^{*}}{\left(1+\Sigma_{+}^{*}\right)(3-2 \gamma)} .
$$

A similar calculation yields the desired expression for $\left\langle\lambda_{\Omega}\right\rangle$.

For $V=1$ (which is an invariant subspace), we have $\rho=1-x^{2}$, and we can rewrite the equation for $\rho$ :

$$
\frac{\left(1-x^{2}\right)^{\prime}}{1-x^{2}}=2 x\left(-3 x \Sigma_{+}^{*}+A^{*}+2 \alpha \cos \theta\right) .
$$

Dividing by $2 x$ and integrating yields $\langle x\rangle=A^{*} / 3 \Sigma_{+}^{*}$ as desired. The expression for $\left\langle\lambda_{\Omega}\right\rangle$ is found analogously.

Now consider the invariant subspace $\rho=0$. This subspace is homeomorphic to the closed interval $[-1,1]$, and hence, no proper periodic orbit can exist there.

The Theorem now follows.

This theorem states exactly when a closed periodic orbit, if it exists, is stable with respect to the variable $\Omega$. Equation (4.9) implies that small deviations from $\Omega=0$ will effectively decay exponentially if $\left\langle\lambda_{\Omega}\right\rangle<0$.

So far we have only given necessary criteria for closed periodic orbits. However, in some cases we can even prove existence of such orbits.

\footnotetext{
${ }^{2}$ By properly periodic we will mean a periodic orbit which is not an equilibrium point. Equilibrium points are trivially periodic in the sense that $c(\tau)=c\left(\tau+T_{n}\right)$ for any $T_{n}$.
} 
Theorem 4.3. For every $\Sigma_{+}^{*} \in(-1,-1 / 4),\left(\lambda^{*}\right)^{2}>1$, and $\gamma \in(0,2)$ there exists one, and only one up to winding number, closed periodic orbit, $\widetilde{\mathcal{E}}\left(V I I_{h}\right)$, with $V=1$ for the dynamical system 4.2).

Proof. Existence: Given $\Sigma_{+}^{*} \in(-1,-1 / 4), \lambda^{2}>1$, and $\gamma \in(0,2)$, we consider the invariant subspace $V=1$. This extremely tilted set is topologically a sphere, $S^{2}$. For $\left(\lambda^{*}\right)^{2}>1$, the variable $\theta$ is strictly monotonic and can thus be considered as a "time" variable. On the sphere $S^{2}$, there are two, and only two, equilibrium points; the North pole, $x=1$ and the South pole, $x=-1$. For $\Sigma_{+}^{*} \in(-1,-1 / 4)$, both of these equilibrium points have two unstable directions, and hence there exist open neighbourhoods $U_{N}$ and $U_{S}$ around the North and South Pole, respectively, such that $S^{2}-\left(U_{N} \cup U_{S}\right)$ is a future trapping region. The compact set $S^{2}-\left(U_{N} \cup U_{S}\right)$ is diffeomorphic to a compact annulus in $\mathbb{R}^{2}$, which allows us to use the Poincaré-Bendixson theorem. This says that there must be either a future attracting equilibrium point, a future attracting closed orbit, or an arbitrary union of points and curves. However, we know there are no other equilibrium points; i.e., there must be a closed curve. Existence is thus proven.

Uniqueness: Since $\theta$ is strictly monotonic, these periodic curves have to be winding around the $x$-axis. Assume there exists two such curves, $c(\theta)$, and $\tilde{c}(\theta)$. Since these two curves cannot cross each other, $\mid x(\theta)-$ $\tilde{x}(\theta) \mid \geq 0$, and equality holds if and only if $c=\tilde{c}$ (up to winding number). By considering the $x$-coordinate for these two closed period orbits, we obtain after a straight-forward manipulation,

$$
|\langle x\rangle-\langle\tilde{x}\rangle| \geq 0,
$$

where equality holds if and only if $c=\tilde{c}$. Using Theorem 4.2 we have $\langle x\rangle=\langle\tilde{x}\rangle$; hence, $c=\tilde{c}$ (up to winding number) and uniqueness is proved.

Theorem 4.4 (Mussel attractor). For $\lambda^{*}=1$ and every $\Sigma_{+}^{*}$ and $\gamma$ taking values in the type IV loophole ( $\left.\gamma_{0}<\gamma<\frac{6}{5+2 \Sigma_{+}^{*}}\right)$ there exists a closed periodic orbit, $\mathcal{C}(I V)$, for the dynamical system 4.2 .

Proof. In this case we can consider the 2-dimensional invariant subspace $\sin \theta=-1$. Again we can use Poincaré-Bendixson theory, however, in a slightly more complicated form. By a consideration of the equilibrium points and the separatrices the existence of a closed curve can be shown (see [25] for details and exact formulation of the theory).

4.1. Stability of the Bianchi type $\mathrm{VII}_{h}$ plane waves. We now return to the general Bianchi type $\mathrm{VII}_{h}$ models. In addition to the analytical results stated above, we have done a comprehensive numerical analysis in the full 7-dimensional state space which is summarised in the Figures to follow. The above results, and from the comprehensive numerical analysis, indicate that the nature of the late-time attractors, as we change the parameter $\lambda^{*}$ from $\lambda^{*}=1$ to $\lambda^{*}>1$, change according to

$\begin{array}{rlll}\mathrm{IV} & \longmapsto & \mathrm{VII}_{h} & \\ \mathcal{L}(I V) & \longmapsto \mathcal{L}\left(V I I_{h}\right) & & \text { (equilibrium point) } \\ \widetilde{\mathcal{L}}_{-}(I V) & \longmapsto \widetilde{\mathcal{L}}\left(V I I_{h}\right) & & \text { (equilibrium point) } \\ \widetilde{\mathcal{F}}_{-}(I V) & \longmapsto \widetilde{\mathcal{F}}\left(V I I_{h}\right) & \text { (closed orbit) } \\ \widetilde{\mathcal{E}}_{-}(I V) & \longmapsto \mathcal{\mathcal { E }}\left(V I I_{h}\right) & \text { (closed orbit) } \\ \mathcal{C}(I V) & \longmapsto \mathcal{T}\left(V I I_{h}\right) & \text { (torus) }\end{array}$

These closed curves, and the torus, are illustrated in Figures 4 and 5 Analysis indicate that the stability of these attractors is preserved under the transition IV $\longmapsto \mathrm{VII}_{h}$. For the curve $\widetilde{\mathcal{E}}\left(V I I_{h}\right)$, this can be shown using similar techniques as above; the region of stabilty in terms of the limiting value $\Sigma_{+}^{*}$ and $\gamma$, is exactly the same as for $\widetilde{\mathcal{E}}_{-}(I V)$ (see Figure 1). 
However, the torus case has to be treated with care; the late-time behaviour is described by a nowhere vanishing flow on a torus. The future behaviour of such integral curves come in two classes according to the nature of the future limit sets [25]:

(1) Rational curves: The attractor is a closed periodic curve which can be described by its homotopy class, $H$, which takes values in the fundamental group of the torus, $\pi_{1}\left(T^{2}\right)=\mathbb{Z} \times \mathbb{Z}$.

(2) Irrational curves: The attractor is an everywhere dense curve on the torus.

The rational curves immediately lend themselves to the analysis above; if $H=(m, n)$, where $n$ is defined as the winding number of $\theta$, then $T_{n}=\frac{n \pi \sqrt{h}}{(1-\langle x\rangle)\left(1+\Sigma_{+}^{*}\right)}$, where $\langle x\rangle$ is given by Theorem 4.2 For an irrational curve, $c(\tau)$, we can utilize the following fact. For any $\tau_{0}$ and $\delta>0$, there exists a closed curve $\tilde{c}(\tau)$ with period $T_{n}$ (not an integral curve) such that $|c(\tau)-\tilde{c}(\tau)|<\delta / 2$ for $\tau_{0}<\tau<\tau_{0}+T_{n}$. In particular, this means that $\left|c\left(\tau_{0}\right)-c\left(\tau_{0}+T_{n}\right)\right|<\delta$, which implies that the limiting curve $c(\tau)$ can be arbitrary close to a closed curve. This implies that we can get arbitrary close to the values given in Theorem 4.2 and hence, we get similar restrictions on the limit cycle. The stability analysis for the variable $\Omega$ can then be applied in a similar manner. Note that for most parameter values in the type $\mathrm{VII}_{h}$ loophole we expect these irrational curves to be attractors. Since the flow on the torus is described continuously by the parameters $\gamma$ and $\Sigma_{+}^{*}$, we would expect that locally the direction of the flow takes all real values in a certain interval. Since the rational numbers only form a set of measure zero we expect that "most" curves will be irrational.

\section{Conclusion}

We have used a dynamical systems approach and a detailed numerical analysis to analyse the late-time behaviour of tilting Bianchi models of types IV and $\mathrm{VII}_{h}$. We established the equations of motion in the ' $N$-gauge' and we found all of the equilibrium points and investigated their stability, with an emphasis on the vacuum plane-wave spacetimes. In particular, it was shown that for $2 / 3<\gamma<2$ there will always be future stable plane-wave solutions in the set of type IV and $\mathrm{VII}_{h}$ tilted Bianchi models and it was proven that the only future attracting equilibrium points for non-inflationary fluids $(\gamma>2 / 3)$ are the plane-wave solutions in Bianchi type $\mathrm{VII}_{h}$ models.

The future asymptotic behaviour of tilted $\gamma$-law perfect fluid models of Bianchi type IV and $\mathrm{VII}_{h}$ can be summarised as follows ${ }^{3}$ :

Bianchi type IV:.

(1) $0<\gamma \leq 2 / 3$ : Asymptotically FRW.

(2) $2 / 3<\gamma<6 / 5$ : Asymptotically a vacuum plane wave. Tilt velocity tends to zero.

(3) $6 / 5<\gamma<4 / 3$ : Asymptotically a vacuum plane wave. Tilt velocity is asymptotically zero, intermediately tilted, intermediately tilted and oscillatory (in the loophole), or extreme.

(4) $4 / 3 \leq \gamma<2$ : Asymptotically a vacuum plane wave. Tilt velocity is asymptotically extreme.

Bianchi type $\mathrm{VII}_{h}$ :

(1) $0<\gamma \leq 2 / 3$ : Asymptotically FRW.

(2) $2 / 3<\gamma<6 / 5$ : Asymptotically a vacuum plane wave. Tilt velocity tends to zero.

(3) $6 / 5<\gamma<4 / 3$ : Asymptotically a vacuum plane wave. Tilt velocity is asymptotically zero, intermediately tilted and oscillatory (either $\widetilde{\mathcal{F}}\left(V I I_{h}\right)$ or the torus attractor), extreme, or extreme and oscillatory.

(4) $4 / 3 \leq \gamma<2$ : Asymptotically a vacuum plane wave. Tilt velocity is asymptotically extreme or extreme and oscillatory.

The stability of the plane-wave solutions is a key result.

\footnotetext{
${ }^{3}$ Models with $0<\gamma<2 / 3$ are subject to the 'no-hair' theorem (originally due to Wald for a cosmological constant 26]). The tilted version was proven in 14 .
} 
FiguRE 4. The figure below displays the dynamical behaviour for values of the parameters near the loophole $\gamma=1.325$ for Bianchi type $\mathrm{VII}_{h}$ non-vacuum tilted models (for the figures $h=1$ is used). When $\Sigma_{+}^{*}=-.2637$, the future asymptotic behaviour is dominated by the stable closed curve $\widetilde{\mathcal{E}}\left(V I I_{h}\right)$. As $\Sigma_{+}^{*}$ increases to a value of $\Sigma_{+}^{*}=-.2578$, the closed loop $\widetilde{\mathcal{E}}\left(V I I_{h}\right)$ becomes unstable (still exists), and a new stable closed loop appears in the form of $\widetilde{\mathcal{F}}\left(V I I_{h}\right)$. When $\Sigma_{+}^{*}=-.2518$, the future asymptotic behaviour is still dominated by the stable closed loop $\widetilde{\mathcal{F}}\left(V I I_{h}\right)$. As $\Sigma_{+}^{*}$ increases to a value of $\Sigma_{+}^{*}=-.2458$, the transverse wrapping of the orbits around the closed loop $\widetilde{\mathcal{F}}\left(V I I_{h}\right)$ is increasing. The orbits wrap transversely around the closed curve $\widetilde{\mathcal{F}}\left(V I I_{h}\right)$ so much so, that they catch up with themselves and create a new stable attractor that is topologically a torus (which we represent as $\left.\mathcal{T}\left(V I I_{h}\right)\right)$. The creation of the torus is not especially clear in this view, because the interior radius of the torus is extremely small. Note the size of the throat of the torus.
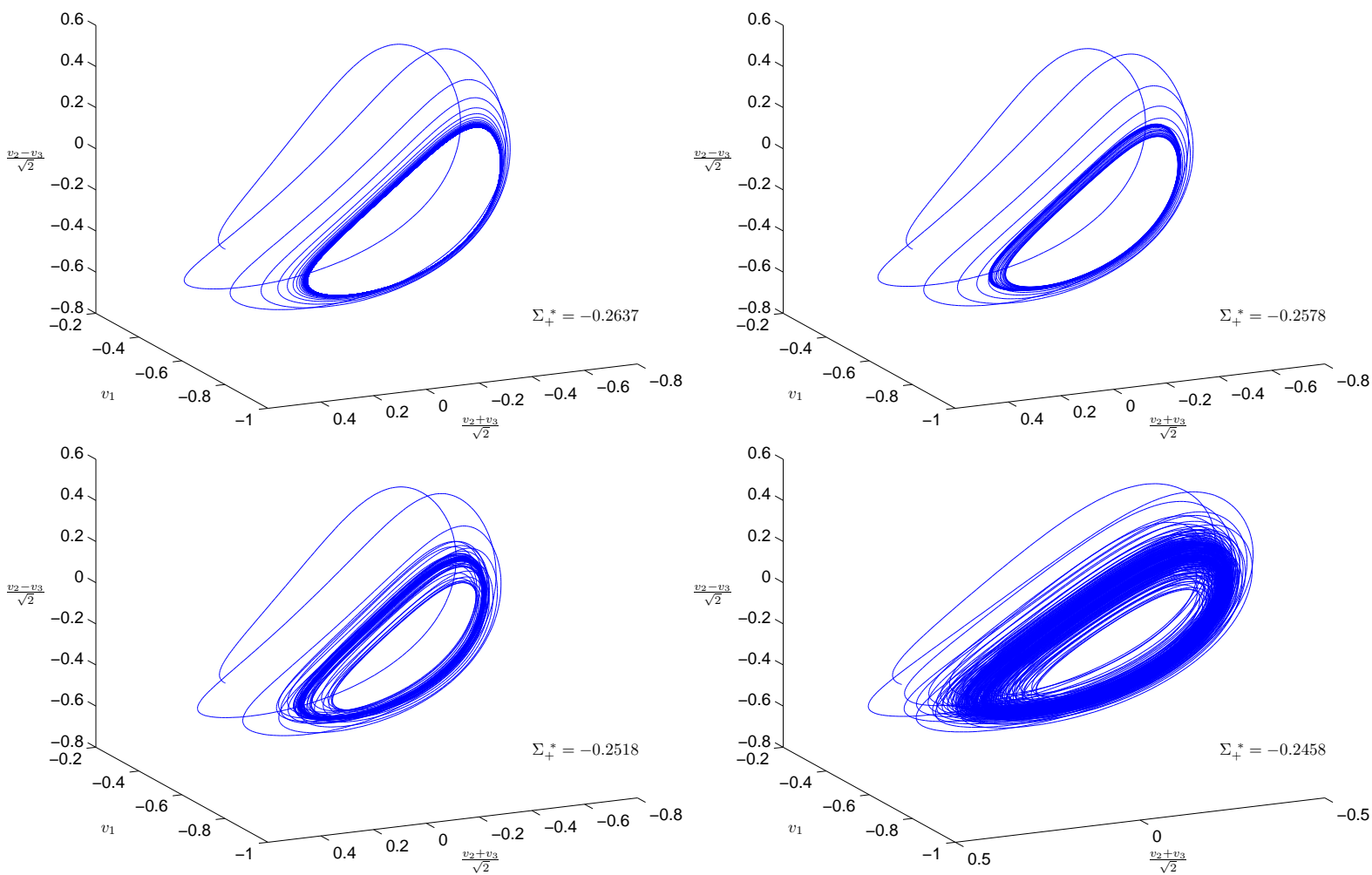

A tiny region of the parameter space (the loophole) was discovered in the Bianchi type IV model which contains no stable plane-wave equilibrium points. We proved the existence of a closed orbit in the loophole and provided criteria for its stability. With the use of theory and extensive numerical experimentation, a limit cycle was found inside the loophole which acts as an attractor (the Mussel attractor).

We then studied the Bianchi type $\mathrm{VII}_{h}$ models numerically. In particular, from a local stability analysis and extensive numerical analysis we found that at late times $\Omega \rightarrow 0$ and the variables 'freeze' into their asymptotic values (in a time scale much shorter than the other dynamical features present). After this freezing has occurred, the system of equations effectively decouples making these models amenable to analytical 
FiguRE 5. The figure below displays the dynamical behaviour for values of the parameters near the loophole $\gamma=1.325$ for Bianchi type $\mathrm{VII}_{h}$ non-vacuum tilted models. As $\Sigma_{+}^{*}$ increases from $\Sigma_{+}^{*}-.2458$ to $\Sigma_{+}^{*}=-.2399$, the interior radius of the torus increases. The closed curve $\widetilde{\mathcal{F}}\left(V I I_{h}\right)$ lies in the interior of the torus. The future asymptotic behaviour is dominated by the attracting torus $\mathcal{T}\left(V I I_{h}\right)$. Note how the throat of the torus narrows as $\Sigma_{+}^{*}$ increases. As $\Sigma_{+}^{*}$ increases from $\Sigma_{+}^{*}-.2339$ to $\Sigma_{+}^{*}=-.2250$, the throat of the torus collapses, and the stable attractor becomes the equilibrium point $\widetilde{\mathcal{L}}_{-}\left(V I I_{h}\right)$.
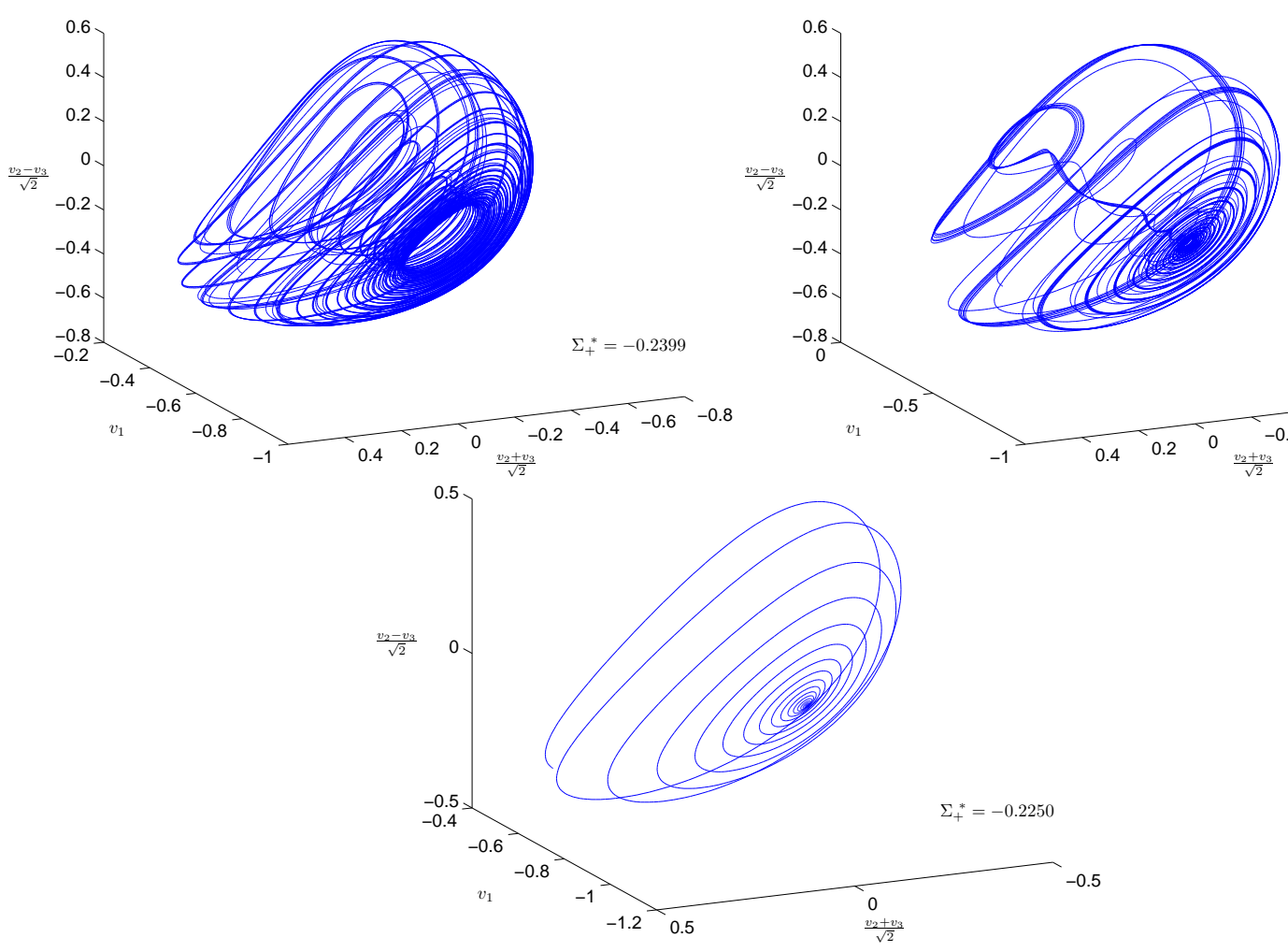

investigations. This, in turn, showed the existence closed curves which interestingly had a characteristic frequency $f=1 / T_{1}=(1-\langle x\rangle)\left(1+\Sigma_{+}^{*}\right) /(\pi \sqrt{h})$. From a detailed numerical analysis of the type $\mathrm{VII}_{h}$ models, we then showed that there is an open set of parameter space in which solution curves approach a compact surface that is topologically a torus. The stability of these late-time attractors seems to be preserved under the transition IV $\longmapsto \mathrm{VII}_{h}$ (e.g., the closed curve in the loophole in the type IV models to the torus in the type $\mathrm{VII}_{h}$ models). Finally, we would like to emphasise that the comprehensive numerical integrations presented in this paper (in the full Bianchi state space) serve to fully justify all of the claims in [14, particularly the 'freezing in' process, the existence of the mussel attractor in the loophole and the existence of the torus.

\section{ACKNOWLEDGMENTS}

This work was supported by NSERC (AC and RvdH) and the Killam Trust and AARMS (SH). 


\section{Appendix A. Numerical Integrations}

\section{A.1. Bianchi type IV and $\mathrm{VII}_{h}$.}

A.1.1. Initial Conditions. The initial conditions are chosen so that the constraint equations are satisfied initially. In both the Bianchi IV and Bianchi $\mathrm{VII}_{h}$ cases the initial conditions are chosen so that $\Sigma_{-}=$ $0.1, \Sigma_{12}=0.01, \Sigma_{13}=0.01, N=0.1, A=0.1$ with $\lambda=1$ for Bianchi type IV, and $\lambda=2 / \sqrt{3}$ for Bianchi type $\mathrm{VII}_{h}$. The initial values for $\Sigma_{+}$and $\Sigma_{23}$ are chosen so that the constraint equations are satisfied. Note how the initial value of $\Sigma_{23}$ changes as a function of $\gamma$.

\begin{tabular}{|c|c|c|c|}
\hline$\Sigma_{+}$ & \multicolumn{3}{|c|}{$\Sigma_{23}$} \\
\hline & $\gamma=1$ & $\gamma=1.325$ & $\gamma=4 / 3$ \\
\hline-0.7 & 0.48980 & 0.43387 & 0.43349 \\
-0.6 & 0.55218 & 0.51171 & 0.51144 \\
-0.5 & 0.59992 & 0.57048 & 0.57028 \\
-0.4 & 0.63632 & 0.61566 & 0.61552 \\
-0.3 & 0.66325 & 0.65009 & 0.65000 \\
-0.2 & 0.68184 & 0.67539 & 0.67534 \\
-0.1 & 0.69275 & 0.69159 & 0.69158 \\
0.0 & 0.69635 & 0.69003 & 0.68999 \\
0.1 & 0.69275 & 0.68016 & 0.68008 \\
0.2 & 0.68184 & 0.66260 & 0.66247 \\
0.3 & 0.66325 & 0.63674 & 0.63657 \\
0.4 & 0.63632 & 0.60154 & 0.60131 \\
0.5 & 0.59992 & 0.55520 & 0.55490 \\
0.6 & 0.55218 & 0.49461 & 0.49422 \\
0.7 & 0.48980 & 0.41357 & 0.41304 \\
\hline
\end{tabular}

A.1.2. Type IV: Numerical Integrations. For completeness we present numerical integrations of the dynamical system for the cosmologically interesting values of $\gamma$ equal to 1 (dust), 1.325 (a dust/radiation mixture) and $4 / 3$ (radiation). Figures 6 to 9 depict some of the results of this integration. The integrations were done over sufficiently long time intervals $[0,800]$, but the plots given here are for time intervals $[0,25]$.

A.1.3. Type $V I I_{h}$ : Numerical Integrations. For completeness we numerically integrate the dynamical system for the cosmologically interesting values of $\gamma$ equal to 1 (dust), 1.325 (a dust/radiation mixture) and 4/3 (radiation). Figures [10 to 13] depict some of the results of this integration. The integrations were done over sufficiently long time intervals $[0,800]$ but the plots given here are for time intervals $[0,25]$ or $[0,100]$ if necessary.

A.2. Bianchi Type $\mathbf{V I I}_{h}, \widetilde{\mathcal{F}}\left(V I I_{h}\right)$ Throat Attractor. Fixing $\gamma=1.325$ and choosing different initial conditions so that the terminal value of $\Sigma_{+}$is 'frozen' in between -0.069773 and -0.025 , we are able to observe some rather interesting dynamics. In this parameter range, we again find that $\widetilde{\mathcal{F}}\left(V I I_{h}\right)$ is a stable attractor. What is perhaps more interesting is the manner in which orbits are attracted to this closed orbit. The attractor $\widetilde{\mathcal{F}}\left(V I I_{h}\right)$ is shown in Figure [14]

A.3. Bianchi Type VII ${ }_{h}, \mathcal{T}\left(V I I_{h}\right)$ Torus Attractor. Figure 15 shows three orbits with initial conditions "far away" from the type $\mathrm{VII}_{h}$ plane wave solutions that approach the torus attractor $\mathcal{T}\left(V I I_{h}\right)$ at late times. This figure illustrates the fact that there exists an open set of initial conditions for which the orbits are attracted to the Bianchi type $\mathrm{VII}_{h}$ loophole. 
Figure 16 depicts the shape and formation of the attractor $\mathcal{T}\left(V I I_{h}\right)$. We choose different time intervals to illustrate the structure of this attractor. We can see how the curve starts to fill up the torus. Irrational curves will eventually completely fill the torus.

\section{REFERENCES}

1. J. Wainwright and G.F.R. Ellis, Dynamical Systems in Cosmology, Cambridge University Press (1997)

2. A.R. King and G.F.R. Ellis, Commun. Math. Phys. 31 (19

3. K.Rosquist and R. T. Jantzen, Phys. Rep. 166 (1988) 89-124.

4. O. I. Bogoyavlensky, (1985) Methods in the Qualitative Theory of Dynamical Systems in Astrophysics and Gas Dynamics Springer-Verlag.

5. J.D. Barrow and D.H. Sonoda, Phys. Reports 139 (1986) 1

6. I.S. Shikin, Sov. Phys. JETP 41 (1976) 794

7. C.B. Collins, Comm. Math. Phys. 39 (1974) 131

8. C. B. Collins and G. F. R.Ellis, Phys.Rep. 56 (1979) 65-105.

9. C.G. Hewitt and J. Wainwright, Phys. Rev. D46 (1992) 4242

10. C.G. Hewitt, R. Bridson, J. Wainwright, Gen.Rel.Grav. 33 (2001) 65

11. J.D. Barrow and S. Hervik, Class. Quantum Grav. 20 (2003) 2841

12. S. Hervik, Class. Quantum Grav. 21 (2004) 2301

13. A. Coley and S. Hervik, Class. Quantum Grav. 21 (2004) 4193-4208

14. A.A. Coley and S. Hervik, Tilted Bianchi models of solvable type, gr-qc/0409100

15. B.J. Carr and A.A. Coley, Class. Quantum Grav. 16 (1999) R31.

16. P. S. Apostolopoulos, gr-qc/0407040

17. J. D. Barrow, Can. J. Phys. 64 (1986) 152

18. J. Wainwright, A.A. Coley, G.F.R. Ellis and M. Hancock, Class. Quantum Grav. 15 (1998) 331.

19. I. D. Novikov, Sov. Astron. 12 (1968) 427

20. C.B Collins and S.W. Hawking, Mon. Not. R. Astron Soc. 162 (1973) 307.

21. A. G. Doroshkevich, V. N. Lukash and I. D. Novikov, Sov. Phys.-JETP 37 (1973) 739.

22. J. D. Barrow, Phys. Rev. D 51 (1995) 3113.

23. J.D. Barrow and C. Tsargas, On the Lukash plane wave solutions

24. R. Tavakol in Dynamical Systems in Cosmology, eds: J. Wainwright and G.F.R. Ellis, Cambridge University Press (1997)

25. S.Kh. Aranson, G.R. Belitsky, E.V. Zhuzhoma, Introduction to the Qualitative Theory of Dynamical Sustems on Surfaces, Trans. Math. Mono. 153 (1996)

26. R.M. Wald, Phys. Rev. D28 (1983) 2118

1 Department of Mathematics \& Statistics, Dalhousie University, Halifax, Nova Scotia, Canada B3H 3J5

2 Department of Mathematics, Statistics and Computer Science, St. Francis Xavier University, Antigonish, Nova Scotia, Canada B2G 2 W5

E-mail address: herviks@mathstat.dal.ca,rvandenh@stfx.ca, aac@mathstat.dal.ca 
Figure 6. Type IV: The figure below displays the future dynamical evolution for $\gamma=1$. The asterisk indicates the initial condition. Note how $\Omega \rightarrow 0$ and $V^{2} \rightarrow 0$ but $\Sigma_{+} \not \rightarrow 0$. In this case, the local sink is $\mathcal{L}(I V)$.
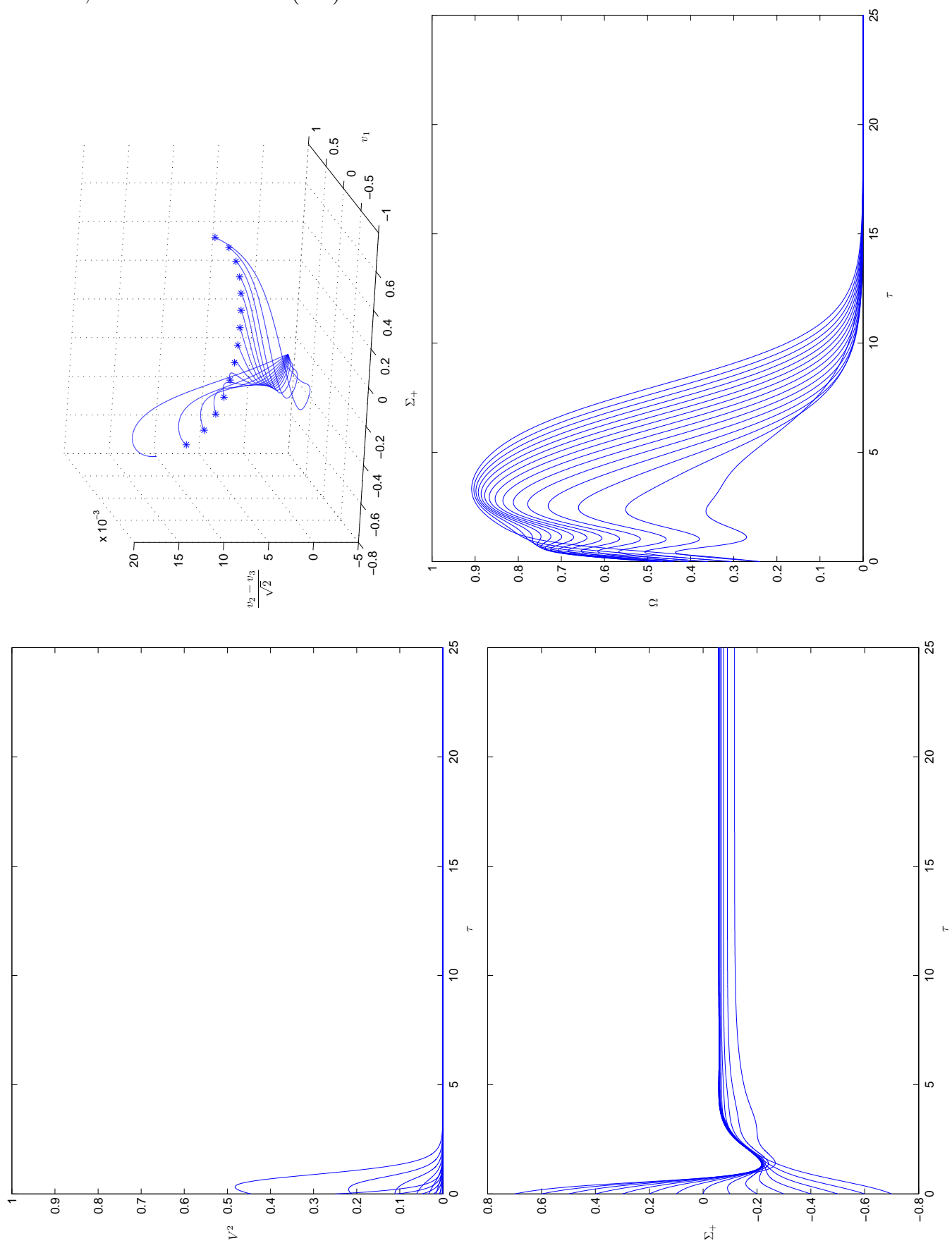
Figure 7. Type IV: The figure below displays the future dynamical evolution for $\gamma=$ 1.325. The asterisk indicates the initial condition. Note how $\Omega \rightarrow 0$ and $V^{2}$ intermediately approaches 1 , but $\Sigma_{+} \not \rightarrow 0$. Also note the timescale.
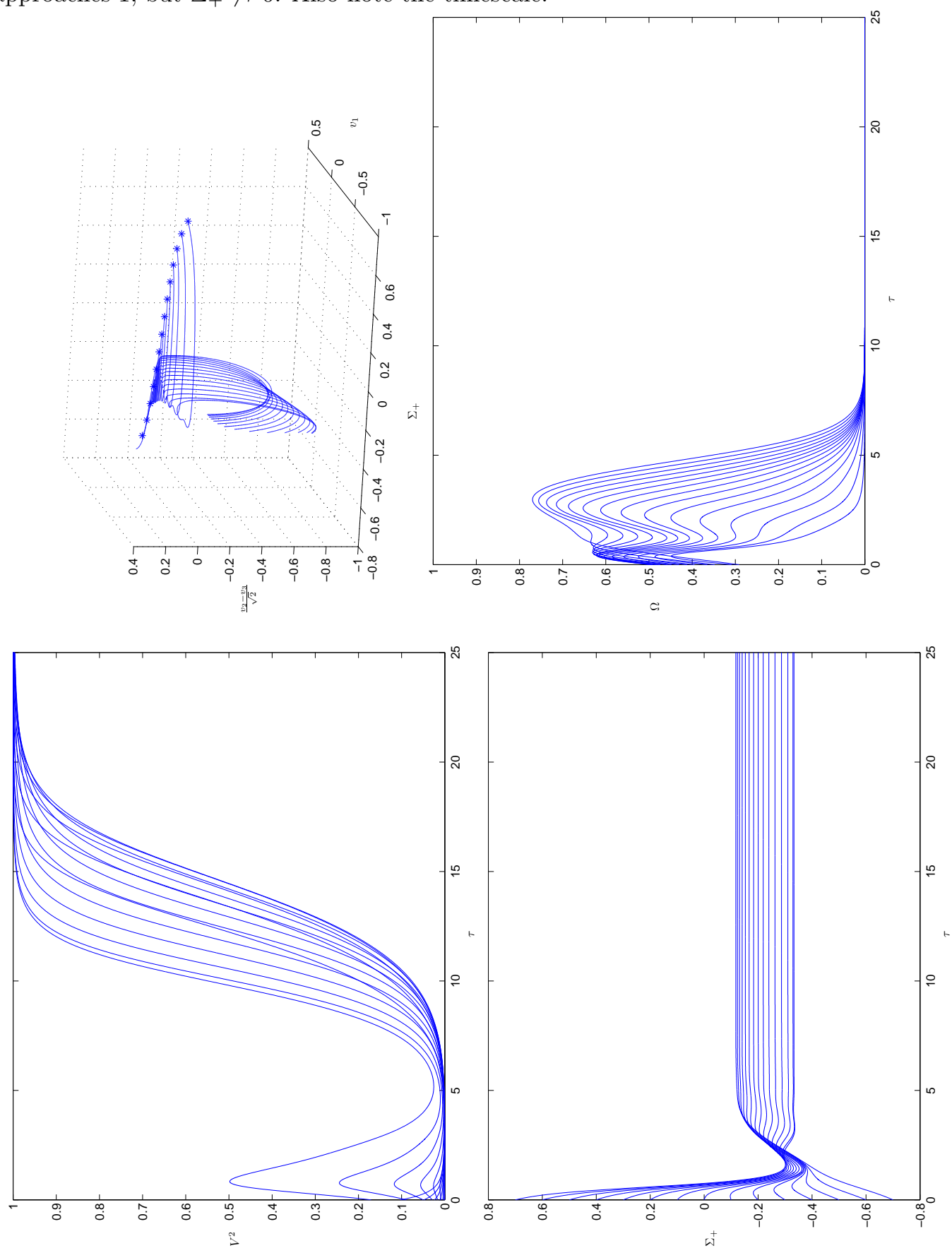
FiguRE 8. Type IV: The figure below displays the future dynamical evolution for $\gamma=1.325$. The timescale has been increased. The asterisk indicates the initial condition. Note how $\Omega \rightarrow 0$ and $V^{2} \rightarrow 1$ or $V^{2}$ oscillates, but $\Sigma_{+} \not \rightarrow 0$. Further analysis of this "exotic" behavior is described in the text. In this case, the local sinks are $\widetilde{\mathcal{E}}(I V), \widetilde{\mathcal{F}}(I V), \widetilde{\mathcal{C}}(I V)$ and $\widetilde{\mathcal{L}}_{-}(I V)$. If we look closely, one closed loop $\mathcal{C}(I V)$ can be seen.
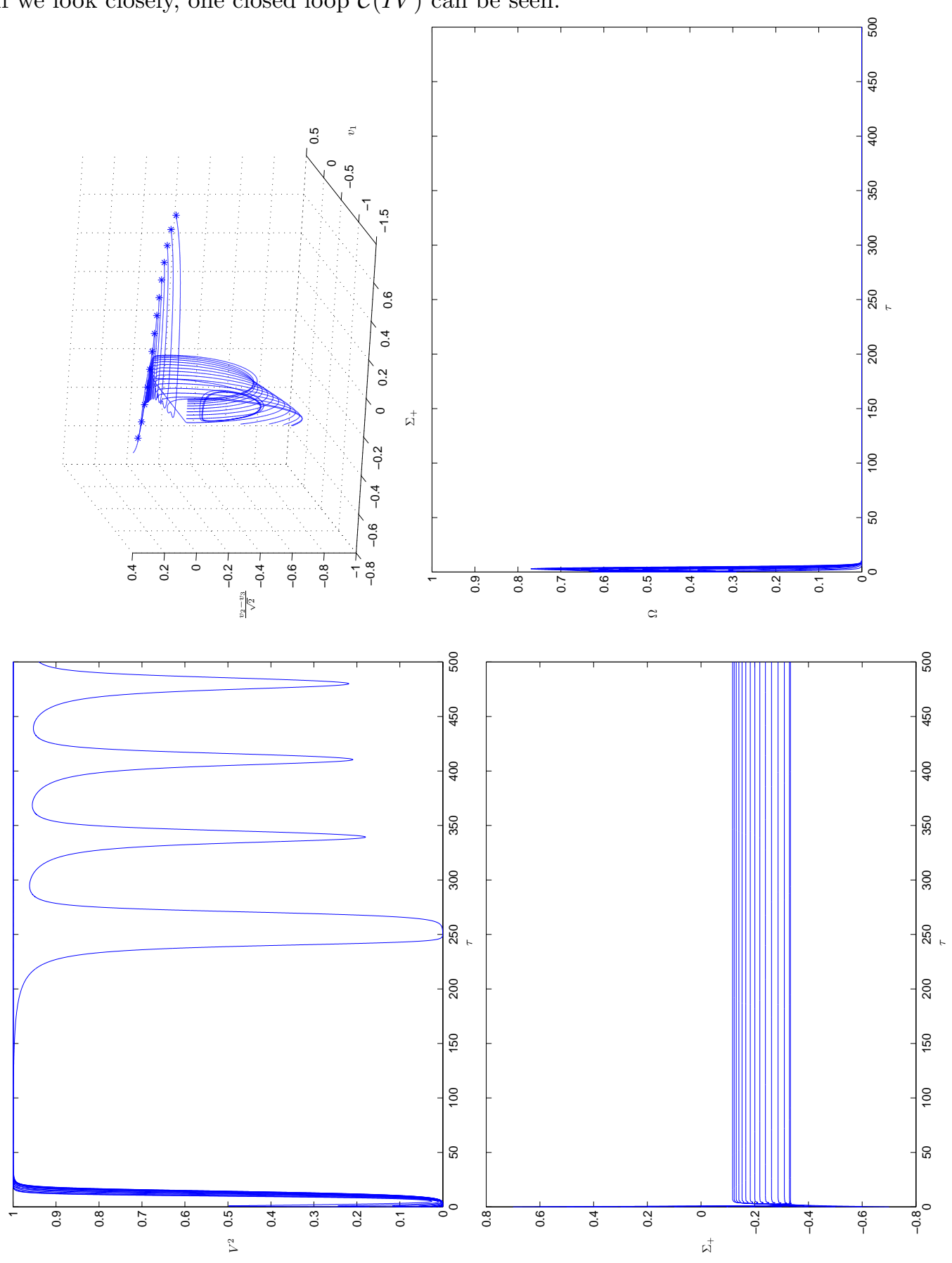
FiguRE 9. Type IV: The figure below displays the future dynamical evolution for $\gamma=4 / 3$. The asterisk indicates the initial condition. Note how $\Omega \rightarrow 0$ and $V^{2} \rightarrow 1$, but $\Sigma_{+} \not \rightarrow 0$. In this case, the local sinks are $\widetilde{\mathcal{E}}(I V)$ and $\widetilde{\mathcal{L}}_{-}(I V)$.
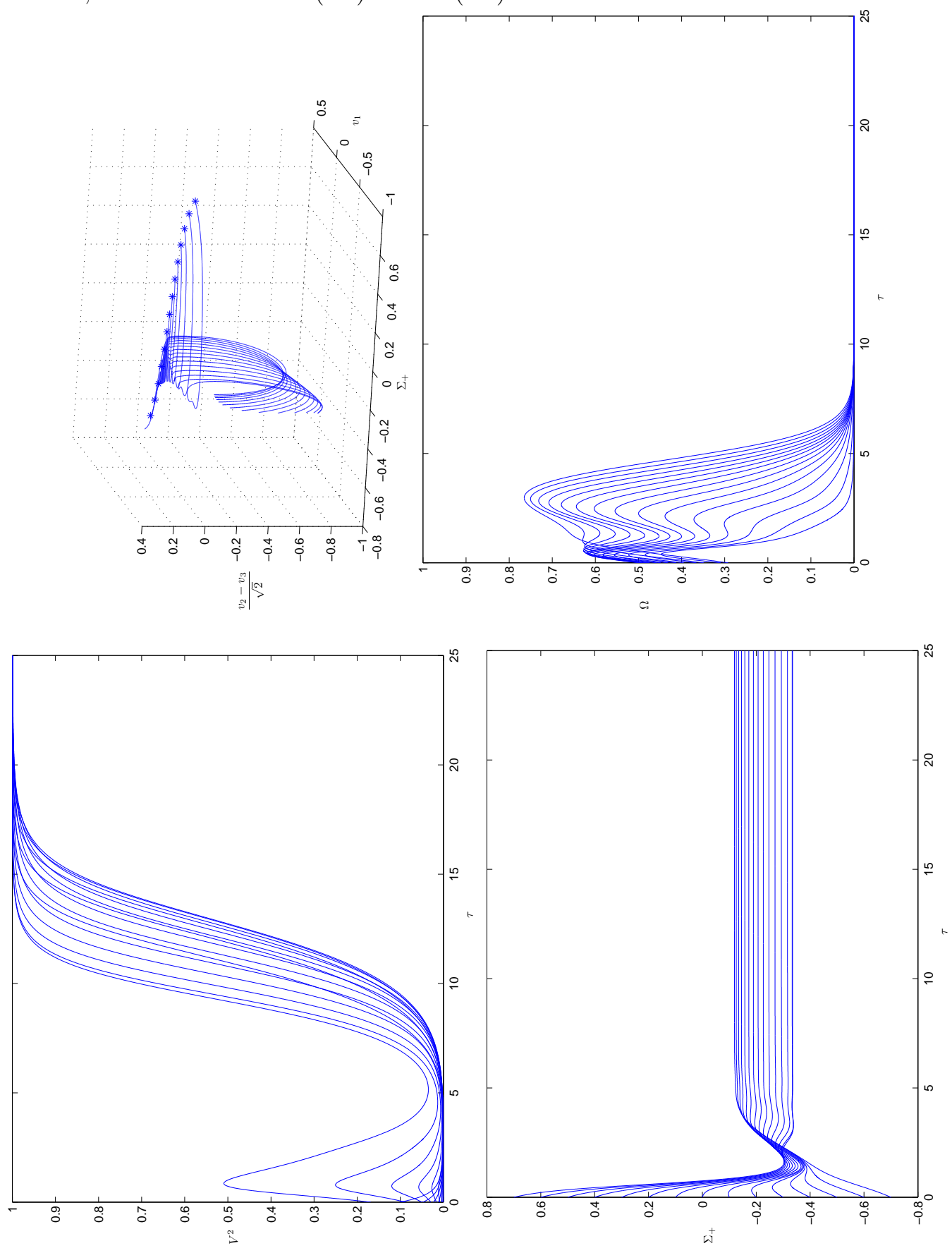
FiguRE 10. Type $\mathrm{VII}_{h}$ : The figure below displays the dynamical behavior for $\gamma=1$. Note how $\Omega \rightarrow 0$ and $V^{2} \rightarrow 0$ but $\Sigma_{+} \not \rightarrow 0$. In this case, the local sink is $\mathcal{L}\left(V I I_{h}\right)$.
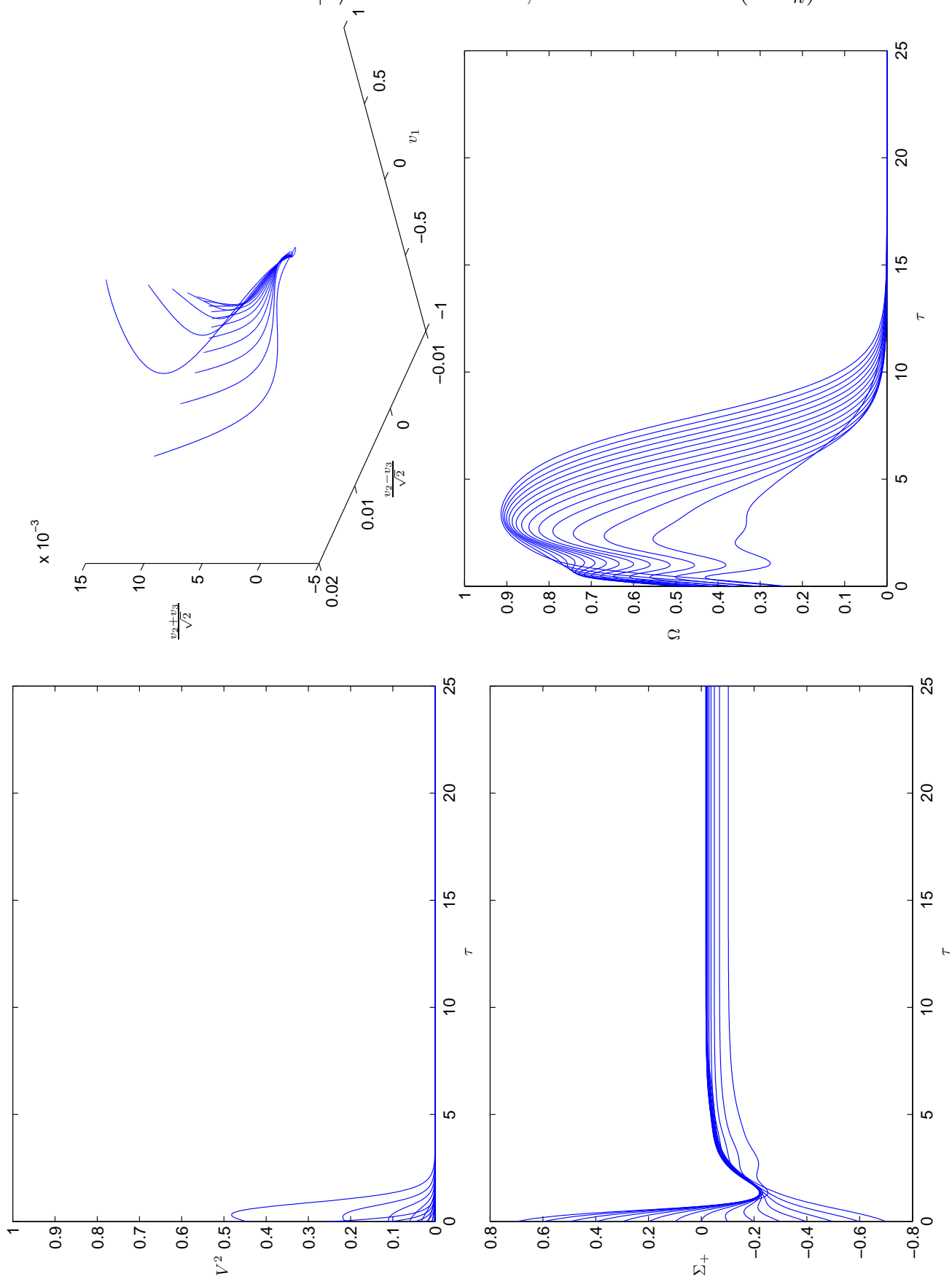
FiguRE 11. Type $\mathrm{VII}_{h}$ : The figure below displays the dynamical behavior for $\gamma=1.325$. Note how $\Omega \rightarrow 0$ but $\Sigma_{+} \not \rightarrow 0$. Note the increased timescale in the graph of $\tau$ vs $V^{2}$. Only two trajectories were sketched in the first figure to show some of the structure of the future asymptotic attractor. Note how both of the trajectories in the first figure spiral into what appears to be a point near $v_{1}=-1$, but then one of the trajectories starts to spiral out again. This is evidence of the torus attractor $\mathcal{T}\left(V I I_{h}\right)$.
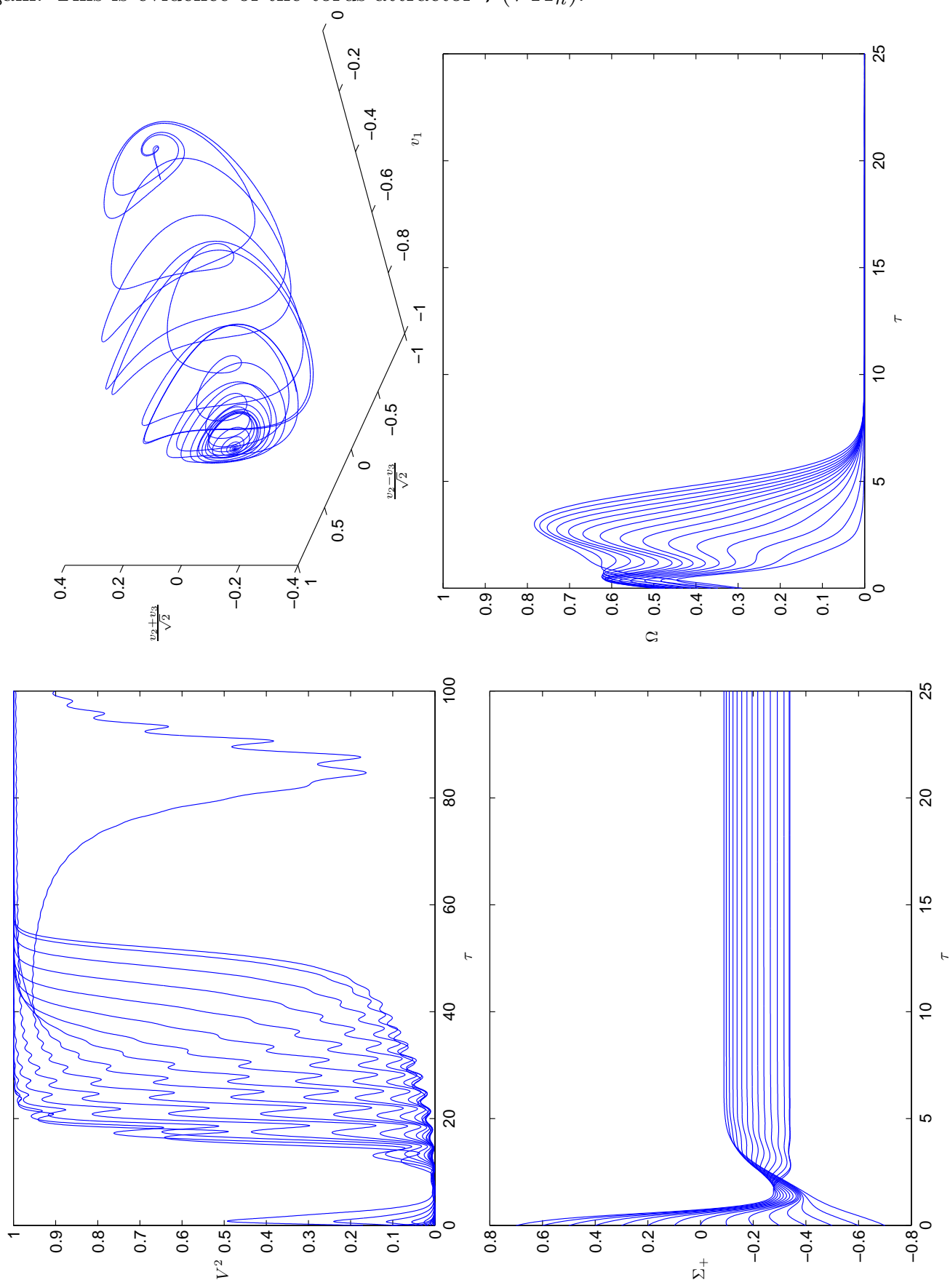
Figure 12. Type $\mathrm{VII}_{h}$ : The figure below displays the dynamical behavior for $\gamma=1.325$. The timescale has been increased in the graph of $\tau$ vs $V^{2}$. Note how $V^{2} \rightarrow 1$ or $V^{2}$ oscillates. Further analysis of this "exotic" behavior takes place in the text. In this case, the local sinks are $\widetilde{\mathcal{E}}\left(V I I_{h}\right), \widetilde{\mathcal{F}}\left(V I I_{h}\right), \mathcal{T}\left(V I I_{h}\right)$ and $\widetilde{\mathcal{L}}_{-}\left(V I I_{h}\right)$. This oscillating behaviour indicates the existence of the attractor $\mathcal{T}\left(V I I_{h}\right)$

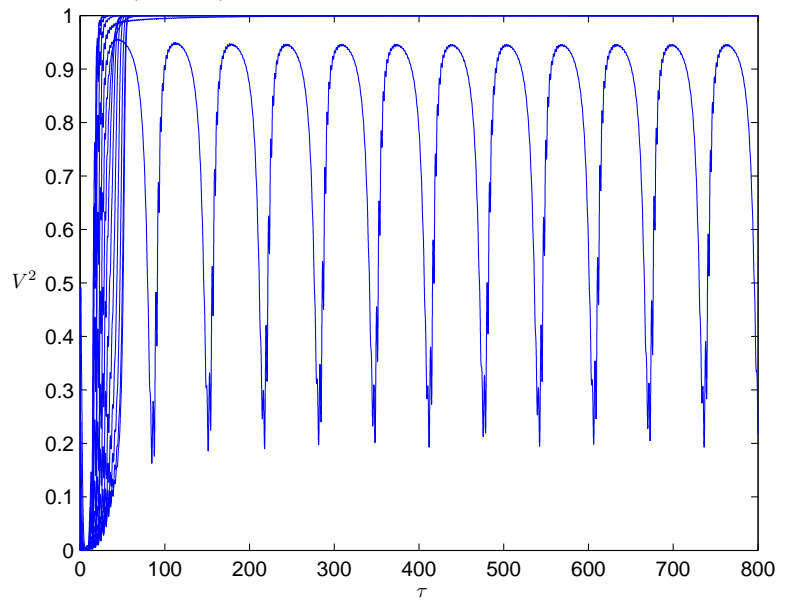


Figure 13. Type $\mathrm{VII}_{h}$ : The figure below displays the dynamical behavior for $\gamma=4 / 3$. Note how $\Omega \rightarrow 0$ and $V^{2} \rightarrow 1$, but $\Sigma_{+} \not \rightarrow 0$. In this case, the local sinks are $\widetilde{\mathcal{E}}\left(V I I_{h}\right)$ and $\widetilde{\mathcal{L}}_{-}\left(V I I_{h}\right)$. Note the increased timescale in the graph of $\tau$ vs $V^{2}$. Only four trajectories were sketched in the first figure to show some of the structure of the future asymptotic attractor.
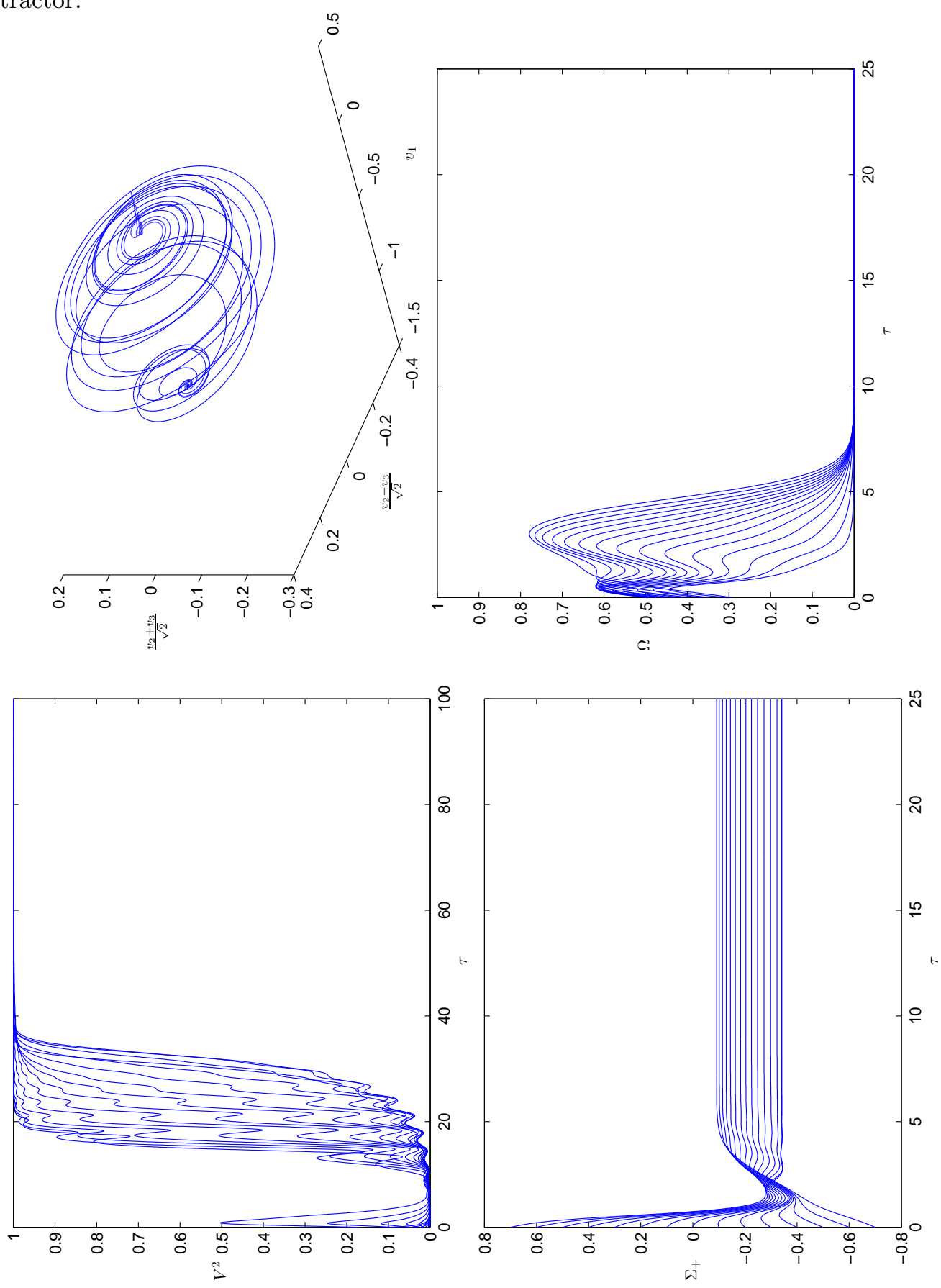
Figure 14. Closed loop $\widetilde{\mathcal{F}}\left(V I I_{h}\right), \Sigma_{+}^{*}=-0.40$. The picture below is magnified to show additional details of the structure.
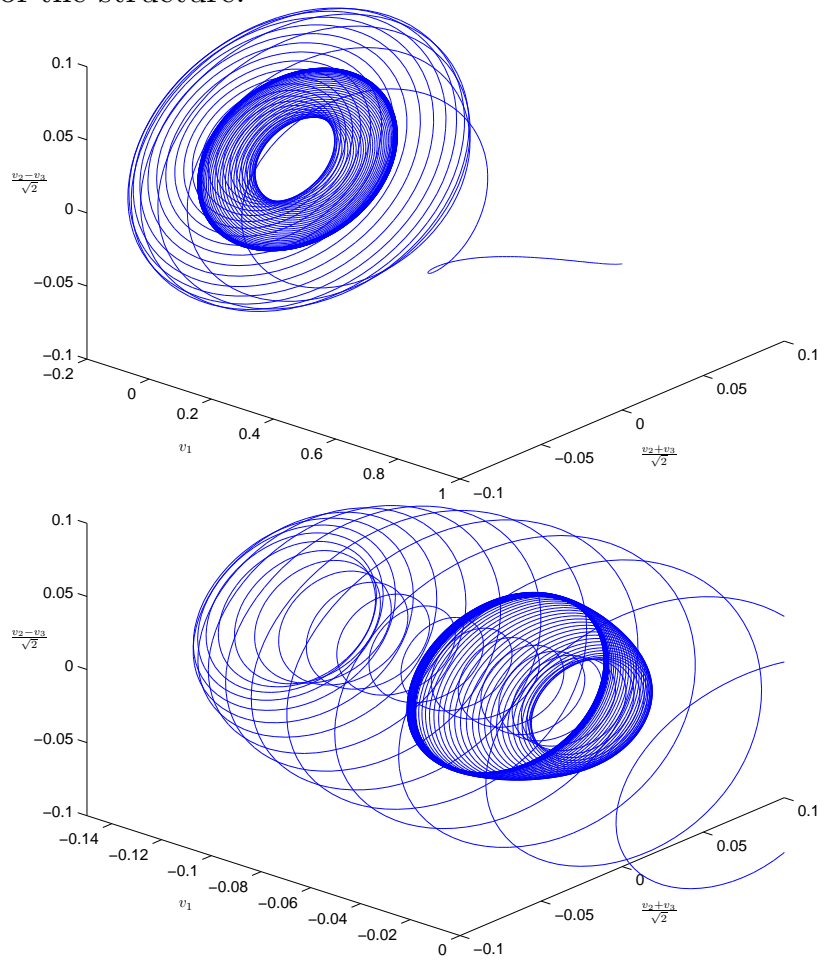

FiguRE 15. The figures below show three orbits with inital conditions "far away" from the plane waves solutions (initial condition is $\left.\left(\Sigma_{+}\right)_{0}=0.2\right)$ that end up in the type $\mathrm{VII}_{h}$ loophole. This illustrates the non-zero measure of the attractor basin of the torus attractor. Note how the orbits start to oscillate after the limiting value $\Sigma_{+}^{*}$ is reached.
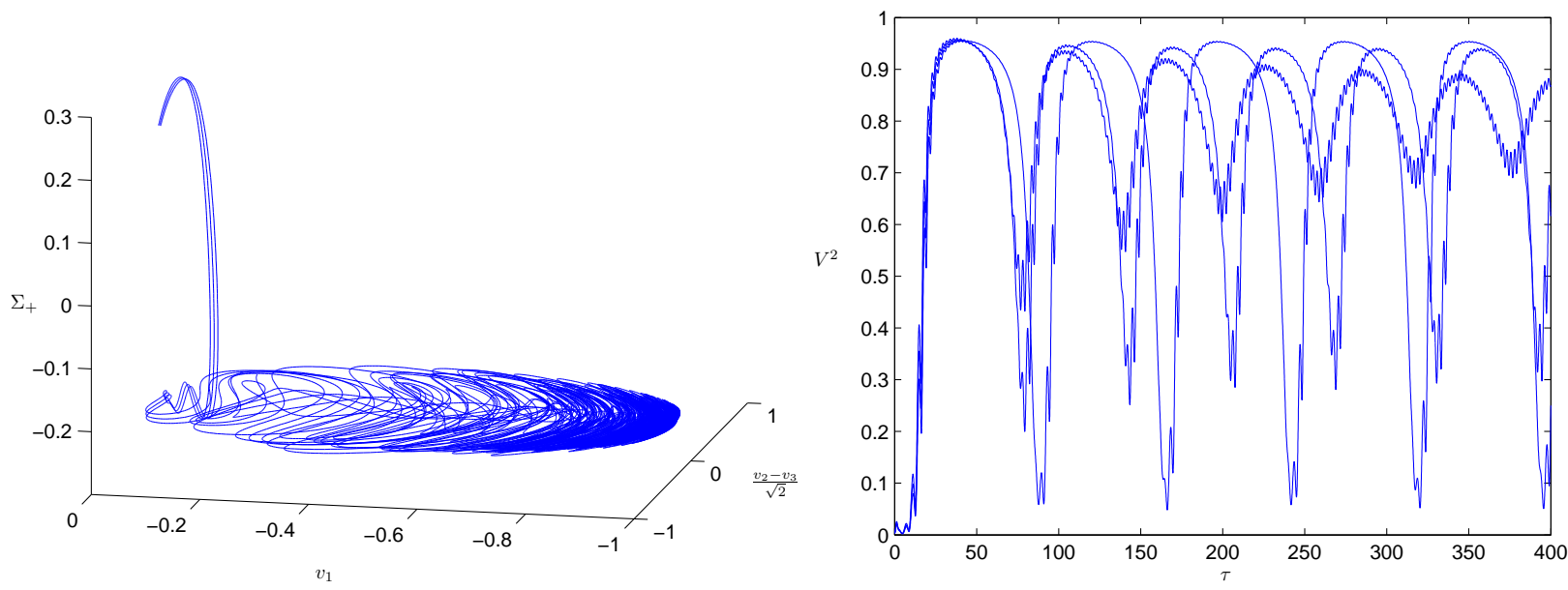

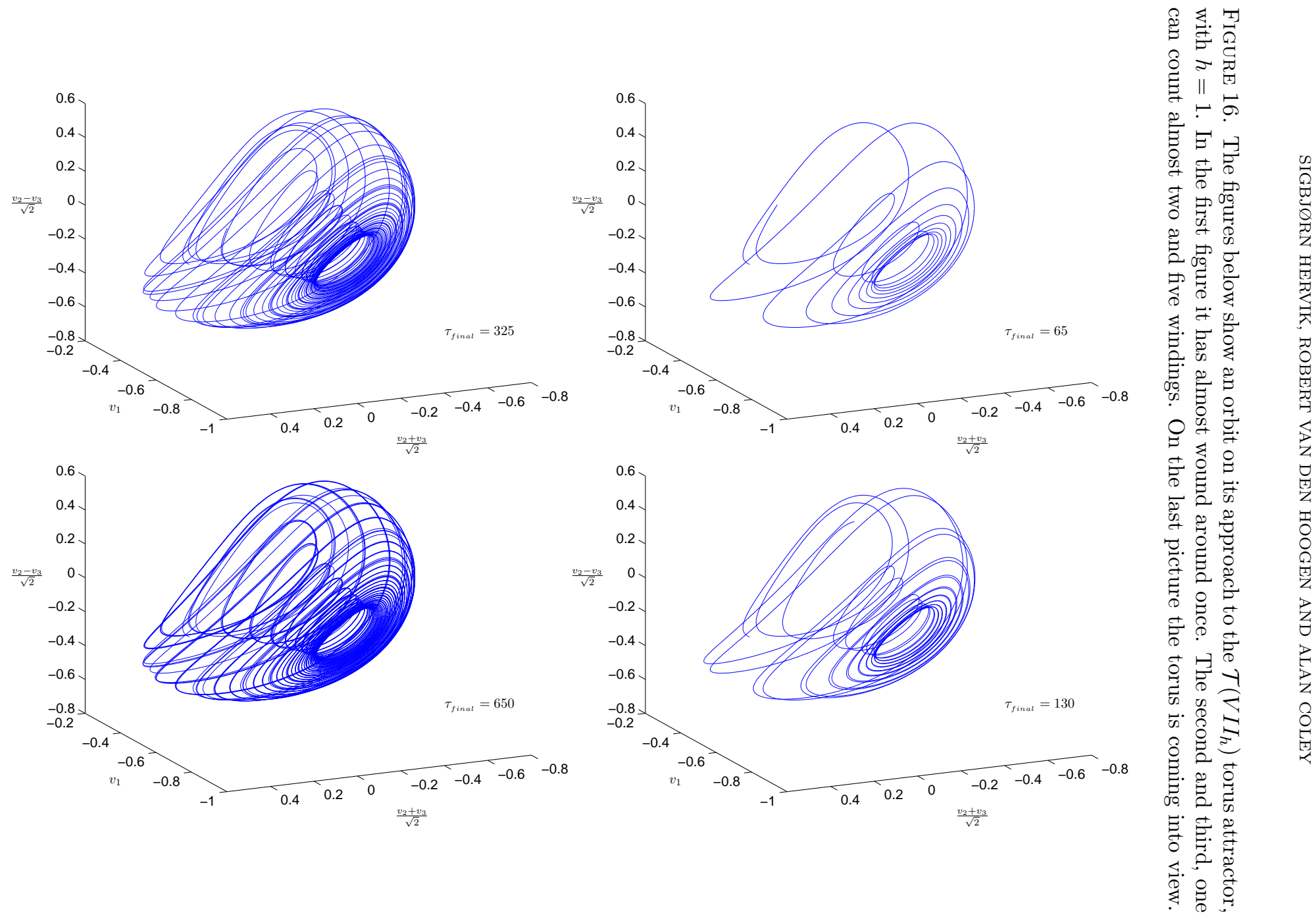